\title{
Effect of Turbulence on Collisional Growth of Cloud Droplets
}

\author{
XIANG-Yu Li, ${ }^{\mathrm{a}, \mathrm{b}, \mathrm{c}, \mathrm{d}, \mathrm{e}, \mathrm{f}}$ AXEL BRANDENBURG, ${ }^{\mathrm{c}, \mathrm{e}, \mathrm{f}, \mathrm{g}, \mathrm{h}}$ GUNILla SVENSSON, ${ }^{\mathrm{a}, \mathrm{b}, \mathrm{d}, \mathrm{i}}$ \\ Nils E. L. HAUGEN, ${ }^{\mathrm{j}, \mathrm{k}}$ BERNHARD MEHLIG, ${ }^{1}$ AND IGOR ROGACHEVSKII ${ }^{\mathrm{m}, \mathrm{c}}$ \\ ${ }^{a}$ Department of Meteorology, Stockholm University, Stockholm, Sweden \\ ${ }^{\mathrm{b}}$ Bolin Centre for Climate Research, Stockholm University, Stockholm, Sweden \\ ${ }^{\mathrm{c}}$ Nordita, KTH Royal Institute of Technology and Stockholm University, Stockholm, Sweden \\ ${ }^{\mathrm{d}}$ Swedish e-Science Research Centre, Stockholm, Sweden \\ ${ }^{\mathrm{e}}$ Laboratory for Atmospheric and Space Physics, University of Colorado Boulder, Boulder, Colorado \\ ${ }^{\mathrm{f}}$ JILA, University of Colorado Boulder, Boulder, Colorado \\ ${ }^{g}$ Department of Astrophysical and Planetary Sciences, University of Colorado Boulder, Boulder, Colorado \\ ${ }^{\mathrm{h}}$ Department of Astronomy, Stockholm University, Stockholm, Sweden \\ ${ }^{\mathrm{i}}$ Global and Climate Dynamics, National Center for Atmospheric Research, Boulder, Colorado \\ ${ }^{\mathrm{j}}$ SINTEF Energy Research, Trondheim, Norway \\ ${ }^{\mathrm{k}}$ Department of Energy and Process Engineering, Norwegian University of Science and Technology, Trondheim, Norway \\ ${ }^{1}$ Department of Physics, Gothenburg University, Gothenburg, Sweden \\ ${ }^{\mathrm{m}}$ Department of Mechanical Engineering, Ben-Gurion University of the Negev, Beer-Sheva, Israel
}

(Manuscript received 11 March 2018, in final form 30 July 2018)

\begin{abstract}
We investigate the effect of turbulence on the collisional growth of micrometer-sized droplets through highresolution numerical simulations with well-resolved Kolmogorov scales, assuming a collision and coalescence efficiency of unity. The droplet dynamics and collisions are approximated using a superparticle approach. In the absence of gravity, we show that the time evolution of the shape of the droplet-size distribution due to turbulence-induced collisions depends strongly on the turbulent energy-dissipation rate $\bar{\varepsilon}$, but only weakly on the Reynolds number. This can be explained through the $\bar{\varepsilon}$ dependence of the mean collision rate described by the Saffman-Turner collision model. Consistent with the Saffman-Turner collision model and its extensions, the collision rate increases as $\bar{\varepsilon}^{1 / 2}$ even when coalescence is invoked. The size distribution exhibits power-law behavior with a slope of -3.7 from a maximum at approximately 10 up to about $40 \mu \mathrm{m}$. When gravity is invoked, turbulence is found to dominate the time evolution of an initially monodisperse droplet distribution at early times. At later times, however, gravity takes over and dominates the collisional growth. We find that the formation of large droplets is very sensitive to the turbulent energy dissipation rate. This is because turbulence enhances the collisional growth between similar-sized droplets at the early stage of raindrop formation. The mean collision rate grows exponentially, which is consistent with the theoretical prediction of the continuous collisional growth even when turbulence-generated collisions are invoked. This consistency only reflects the mean effect of turbulence on collisional growth.
\end{abstract}

\section{Introduction}

Collisional growth of inertial particles in a turbulent environment plays an important role in many physical processes (Pumir and Wilkinson 2016; Ohno and Okuzumi 2017). For instance, collisional growth of droplets in atmospheric clouds may explain the rapid warm rain formation (Shaw 2003; Devenish et al. 2012; Grabowski and Wang 2013). Collisions of dust grains in circumstellar disks is proposed to be a key step toward planet formation (Johansen and Lambrechts 2017).

Corresponding author: Xiang-Yu Li, xiang.yu.li@su.se
The most notorious difficulty is how turbulence affects the collisional growth. This problem has a long history and was recently reviewed by Shaw (2003), Devenish et al. (2012), Grabowski and Wang (2013), and Pumir and Wilkinson (2016). The pioneering work by Saffman and Turner (1956) proposed a theoretical model for the collision rate (Saffman-Turner model) of cloud droplets. The key idea of the Saffman-Turner model is that the collision rate is dominated by small scales of turbulence since the size of cloud droplets (typical size is $10 \mu \mathrm{m}$ in radius) is three orders of magnitude smaller than the Kolmogorov length (i.e., the smallest scale of turbulence, which is about $1 \mathrm{~mm}$ in atmospheric clouds). 
The Saffman-Turner model predicts that the mean collision rate $\bar{R}_{c}$ is proportional to the mean energy dissipation rate $\bar{\varepsilon}$ if there is no intermittency and the particle inertia is small. Following Saffman and Turner (1956), Reuter et al. (1988) used a stochastic model to show that turbulence fluctuations modeled by random perturbations enhance the collision rate. Grover and Pruppacher (1985) studied the effect of vertical turbulent fluctuations on the collision between aerosol particles and cloud droplets using a one-dimensional model. They inferred that three-dimensional atmospheric turbulence should cause a substantial enhancement of the collision rate. The stochastic model developed by Pinsky and Khain (2004) demonstrated that the turbulence enhancement can reach a factor of up to 5 . Follow-up studies of Pinsky et al. (2007, 2008) showed that turbulence has a significant enhancement effect on the collision rate, especially for small and similar-sized droplets (radius of a few micrometers). However, Koziol and Leighton (1996) found that, using a stochastic model, turbulence only has a moderate effect on the collision rate. This discrepancy between the two stochastic models is either due to the simplified descriptions of the droplet motion or an inaccurate modeling of turbulence fluctuations (Wang et al. 2005; Grabowski and Wang 2013).

Because of the rapid advances in computer technology, the collision rate has been studied using direct numerical simulations (DNSs). Most of the DNS studies focused on the collisional growth without coalescence. Such studies are useful in that they facilitate our understanding of the physical mechanisms contributing to the collision rate, such as droplet clustering and relative velocity. Sundaram and Collins (1997b) constructed the collision rate using the radial distribution velocity and the radial distribution function based on the SaffmanTurner collision rate. Their subsequent work found that turbulence has a significant effect on droplet clustering and on the relative velocity, which demonstrated its importance for the collision rate (Shaw et al. 1998; Collins and Keswani 2004; Chun et al. 2005a; Salazar et al. 2008; Ireland et al. 2016a,b). The turbulence effect on the geometrical collisional kernel was investigated by Franklin et al. (2005), Ayala et al. (2008a), Rosa et al. (2013), and Chen et al. (2016). Ayala et al. (2008b) developed a comprehensive parameterization of the turbulent collision rate and concluded that turbulence increases the geometrical collision rate by up to $47 \%$ with increasing energy dissipation rate. They also found that the dependence of the collision rate on the Reynolds number is minor. Rosa et al. (2013) and Chen et al. (2016) confirmed the secondary dependency of the collision statistics on the Reynolds number.
Invoking coalescence is computationally and technically more demanding, but more realistic. Riemer and Wexler (2005) found that the turbulent collision rate is several orders of magnitude larger than the gravitational collision rate. However, Wang et al. (2006) argued that this result is grossly overestimated because their rms velocity was overestimated by a factor of $\sqrt{3}$ and their treatment of the collision efficiency only included gravity but not turbulence. Franklin (2008) investigated collision-coalescence processes by solving the Smoluchowski equation together with the NavierStokes equation using DNS and found that the size distribution of cloud droplets is significantly enhanced by turbulence. Using a similar approach, Xue et al. (2008) concluded that even a moderate turbulence enhancement of the collision rate can have a significant effect on the growth of similar-sized droplets, which is referred to as the autoconversion phase of the growth. A similar conclusion was reached by Wang and Grabowski (2009), who found that turbulence enhances the collisional growth by a factor of 2 . They also found that in their simulations the dependence on Reynolds number is uncertain due to its small value. Onishi and Seifert (2016) updated the collision rate model of Wang and Grabowski (2009) and performed DNS at higher Reynolds number. They found that the collisional growth of cloud droplets depends on the Reynolds number. However, they did not study the time evolution of the size distribution nor its dependency on Reynolds number and energy dissipation rate. Chen et al. (2018) investigated the time evolution of the size distribution and its dependence on the energy dissipation rate and Reynolds number using a Lagrangian collision-detection method. They concluded that turbulence has a prominent effect on the broadening of the size distributioneven if the turbulence intensity is small. However, a comparison between the theoretical predictions of the $\bar{\varepsilon}$ dependence of the collision rate (Saffman and Turner 1956) and the numerical simulations results was not performed for the case when coalescence was invoked. Also, the effect of the initial width of the size distribution on the turbulence enhancement was not discussed.

In fully developed turbulence, droplet collisioncoalescence process results in a wide range of droplet sizes and thus in a wide range of droplet Stokes numbers that evolve during the simulation. The Stokes number is a dimensionless measure of the effect of droplet inertia, which depends on the geometrical droplet size and the turbulence intensity. In cloud turbulence with $\bar{\varepsilon} \approx 0.04 \mathrm{~m}^{2} \mathrm{~s}^{-3}$, the Stokes number (St) varies from $10^{-3}$ (droplet radius of about $1 \mu \mathrm{m}$ ) to 10 (about $100 \mu \mathrm{m}$ ) and beyond. Very small cloud droplets (for $\mathrm{St} \ll 1$ ) are advected by turbulent airflow and the collision is caused 
by local turbulent shear (Saffman and Turner 1956; Andersson et al. 2007). For larger Stokes numbers, on the other hand, inertial effects become important, which allow the droplets to detach from the flow. This may substantially increase the collision rate (Sundaram and Collins 1997a; Falkovich et al. 2002; Wilkinson et al. 2006). The time-dependent collision rate due to the dynamical Stokes number cannot be captured with a predetermined parameterization of the collision rate. Saito and Gotoh (2018) developed a Lagrangian algorithm to detect collisions without using a parameterized collision kernel. They observed that turbulence broadens the size distribution of cloud droplets. Since their work has condensation included, it is unclear if the broadening of the size distribution results from the turbulence effect on the collisions or its effect on condensation. Such a broadening of the size distribution due to condensation could result from turbulence-facilitated supersaturation fluctuations (Sardina et al. 2015).

To quantify the role of small-scale turbulence on the time evolution of the size distribution and its connection to the Saffman-Turner collision rate (Saffman and Turner 1956) when coalescence is included, we investigate the collisional growth of cloud droplets with or without gravity. We determine the droplet-size distribution directly from numerical simulations, thus avoiding the use of a parameterized kernel. We focus on the time evolution of the size distribution due to collisioncoalescence processes and how changing the Reynolds number and the energy dissipation rate affect the size distribution. We perform high-resolution DNS of turbulence with a well-resolved Kolmogorov viscous scale (our maximum Taylor-microscale Reynolds number is 158). Droplet and collision dynamics are solved together using a superparticle approach assuming unit collision and coalescence efficiency. Unit coalescence efficiency means that droplets coalesce upon collision. Compared with the direct Lagrangian collision-detection method, the superparticle approach is computationally less demanding. This can be deduced from a cross comparison with the Eulerian approach. First, Li et al. (2017) found that the superparticle is about 10 times faster than an Eulerian approach where one solves the Smoluchowski and momentum equations for logarithmically spaced mass bins. Second, the direct Lagrangian collisiondetection method is more costly than the Eulerian approach (Chen et al. 2018). Therefore, the superparticle approach is at least 10 times faster than the direct Lagrangian collision-detection method. More importantly, the superparticle approach can easily be extended to large-eddy simulations with an appropriate subgrid-scale model (Grabowski and Abade 2017). A detailed comparison of the present simulation results and the theoretical prediction of the collision rate (Saffman and Turner 1956) is conducted. In addition, we explore how the width of the initial size distribution alters the turbulence effect on collisional growth of cloud droplets. In the meteorology community, the process of collision and coalescence is referred to as collection, while in the astrophysical community, this process is referred to as coagulation. Since we assume unit coalescence efficiency in the present study, we use the terminology collision. To address the turbulencefacilitated collision for more general applications (such as interstellar dust), we will first focus on DNS without gravity. We will then turn to DNS with both gravity and turbulence, which is important for the cloud droplet formation.

\section{Numerical setup}

Our simulations are conducted using the Pencil Code. The DNS of the turbulent flow are performed for a weakly compressible gas, and we adopt a superparticle algorithm to approximate the droplet dynamics (Zsom and Dullemond 2008; Shima et al. 2009; Johansen et al. 2012).

\section{a. DNS of the turbulent airflow}

The velocity $\mathbf{u}$ of the turbulent airflow is determined by the Navier-Stokes equation:

$$
\frac{\partial \mathbf{u}}{\partial t}+\mathbf{u} \cdot \nabla \mathbf{u}=\mathbf{f}-\rho^{-1} \nabla p+\rho^{-1} \nabla \cdot(2 \nu \rho S),
$$

where $\mathbf{f}$ is a monochromatic random forcing function (Brandenburg 2001), $\nu$ is the kinematic viscosity of the airflow, $S_{i j}=(1 / 2)\left(\partial_{j} u_{i}+\partial_{i} u_{j}\right)-(1 / 3) \delta_{i j} \nabla \cdot \mathbf{u}$ is the traceless rate-of-strain tensor, $p$ is the gas pressure, and $\rho$ is the gas density, which in turn obeys the continuity equation,

$$
\frac{\partial \rho}{\partial t}+\nabla \cdot(\rho \mathbf{u})=0
$$

We assume that the gas is isothermal with constant sound speed $c_{s}$, so that $c_{s}^{2}=\gamma p / \rho$, where $\gamma=c_{P} / c_{V}=7 / 5$ is the ratio between specific heats, $c_{P}$ and $c_{V}$, at constant pressure and constant volume, respectively. To avoid global transpose operations associated with calculating Fourier transforms for solving the nonlocal equation for the pressure in strictly incompressible calculations, we solve here instead the compressible Navier-Stokes equations using high-order finite differences. To simulate the nearly incompressible atmospheric airflow, we set the sound speed to $5 \mathrm{~m} \mathrm{~s}^{-1}$, resulting in a Mach number of 0.06 when the $u_{\mathrm{rms}}=0.27 \mathrm{~m} \mathrm{~s}^{-1}$. Such a 
TABLE 1 . Summary of the simulations, with $f_{0}$ the amplitude of the random forcing (see text) and $L$ the domain size.

\begin{tabular}{lccllclrrr}
\hline \hline Run & $N_{p} / 10^{6}$ & $N_{\text {grid }}$ & \multicolumn{1}{c}{$f_{0}$} & $L(\mathrm{~m})$ & $u_{\mathrm{rms}}\left(\mathrm{m} \mathrm{s}^{-1}\right)$ & $\mathrm{Re}_{\lambda}$ & $\bar{\varepsilon}\left(\mathrm{m}^{2} \mathrm{~s}^{-3}\right)$ & $\eta \times 10^{-4}(\mathrm{~m})$ & $\tau_{\eta}(\mathrm{s})$ \\
\hline A & 8.4 & $256^{3}$ & 0.02 & 0.125 & 0.17 & 57 & 0.039 & 4 \\
B & 67 & $512^{3}$ & 0.02 & 0.25 & 0.21 & 94 & 0.04 & 4 \\
C & 67 & $512^{3}$ & 0.02 & 0.50 & 0.27 & 158 & 0.036 & 0.016 \\
D & 67 & $512^{3}$ & 0.0072 & 0.44 & 0.13 & 98 & 0.005 & 4 \\
E & 67 & $512^{3}$ & 0.01 & 0.37 & 0.15 & 97 & 0.01 & 7 & 0.017 \\
F & 67 & $512^{3}$ & 0.014 & 0.30 & 0.18 & 94 & 0.02 & 0.044 \\
\hline
\end{tabular}

configuration with so small Mach number is equivalent to an incompressible flow. Indeed, we quantify the weak compressibility in our DNS by calculating the dimensionless number $\wp=\left\langle|\nabla \cdot \mathbf{u}|^{2}\right\rangle /\left\langle|\nabla \times \mathbf{u}|^{2}\right\rangle=2 \times 10^{-4}$. Following Gustavsson and Mehlig (2016b), the parameter $\wp=2 \times 10^{-4}$ corresponds to a Stokes number $\mathrm{St}=0.018$. The smallest Stokes number in our DNS is $\mathrm{St}=0.05$. This implies that the effect of fluid compressibility is much less than the compressibility of the particle velocity field caused by droplet inertia. Therefore, the effect of fluid compressibility on the droplets is also negligible.

To characterize the intensity of turbulence, we use the Taylor microscale Reynolds number $\operatorname{Re}_{\lambda} \equiv u_{\mathrm{rms}}^{2} \sqrt{5 /(3 \nu \bar{\varepsilon})}$, where $u_{\mathrm{rms}}$ is the rms turbulent velocity, and $\bar{\varepsilon}=2 \nu \overline{\operatorname{Tr} S_{i j} S_{j i}}$ is the mean energy-dissipation rate per unit mass and $\operatorname{Tr}$ denotes the trace. The parameters of all simulations are listed in Table 1 . Here $\tau_{\eta}=(\nu / \bar{\varepsilon})^{1 / 2}$ is the Kolmogorov time and $\eta=\left(\nu^{3} / \bar{\varepsilon}\right)^{1 / 4}$ is the Kolmogorov length.

\section{b. Superparticle algorithm}

The equations governing the dynamics and collision of droplets in a turbulent flow are solved simultaneously with the Navier-Stokes equations. We approximate the droplet dynamics and collisions using a stochastic Monte Carlo algorithm (Bird 1978, 1981; Jorgensen et al. 1983) that represents a number of spherical droplets by a superparticle (Zsom and Dullemond 2008; Shima et al. 2009; Johansen et al. 2012; Li et al. 2017). All droplets in superparticle $i$ are assumed to have the same material density $\rho_{d}$, radius $r_{i}$, and velocity $v_{i}$. Further, each superparticle is assigned a volume of the grid cell and thus a droplet number density $n_{i}$. The position $\mathbf{x}_{i}$ of superparticle $i$ is determined by

$$
\frac{d \mathbf{x}_{i}}{d t}=\mathbf{V}_{i}
$$

and

$$
\frac{d \mathbf{V}_{i}}{d t}=\frac{1}{\tau_{i}}\left(\mathbf{u}-\mathbf{V}_{i}\right)+\mathbf{g} .
$$

Here,

$$
\tau_{i}=2 \rho_{d} r_{i}^{2} /\left[9 \rho \nu D\left(\mathrm{Re}_{i}\right)\right]
$$

is the particle response time attributed to superparticle $i$. The correction factor (Schiller and Naumann 1933; Marchioli et al. 2008)

$$
D\left(\operatorname{Re}_{i}\right)=1+0.15 \operatorname{Re}_{i}^{2 / 3}
$$

models the effect of nonzero particle Reynolds number $\mathrm{Re}_{i}=2 r_{i}\left|\mathbf{u}-\mathbf{V}_{i}\right| / \nu$. This is a widely used approximation, although it does not correctly reproduce the small- $\mathrm{Re}_{i}$ correction to Stokes formula (Veysey and Goldenfeld 2007). The dimensionless particle-response time is given by the Stokes number $\mathrm{St}=\tau_{i} / \tau_{\eta}$. Droplets are randomly distributed in the simulation domain with zero velocity initially. The term $\mathbf{g}$ in Eq. (4) is included only when collisions are also driven by gravity, in addition to turbulence.

Droplet collisions are represented by collisions of superparticles (Shima et al. 2009; Johansen et al. 2012; Li et al. 2017). When two superparticles collide, two droplets in either of the superparticles can collide with probability $p_{c}=\tau_{c}^{-1} \Delta t$, where $\Delta t$ is the integration time step. A collision event occurs when $p_{c}>\eta_{c}$, where $\eta_{c}$ is a random number. If a collision event happens, $\eta_{c}$ must lie between zero and one; see appendix A for details on the collision scheme. A mean-field model is adopted for the collision time $\tau_{c}$ :

$$
\tau_{c}^{-1}=\sigma_{c} n_{j}\left|\mathbf{V}_{i}-\mathbf{V}_{j}\right| E_{c} .
$$

Here $\sigma_{c}=\pi\left(r_{i}+r_{j}\right)^{2}$ is the geometric collision cross section between two droplets with radii $r_{i}$ and $r_{j}$. The parameter $E_{c}$ is the collision efficiency (Devenish et al. 2012). It is set to unity in our simulations, and we assume that droplets coalesce upon collision. The use of a unit collision efficiency overestimates the collision rate. This is done to reduce the complexity of the simulation and enables us to focus only on the collision dynamics. Adopting unit coalescence efficiency is justified by the fact that the Weber number is only about $10^{-2}$ (Perrin and Jonker 2015). The Weber number is defined as the ratio of droplet inertia and its surface tension (Perrin and Jonker 2015). A low Weber number means that the 
colliding droplets always coalesce. It is worth noting that a cylindrical kernel is used in the present superparticle scheme as described by Eq. (7). This is in contrast to what is done for Lagrangian point particle simulations (Wang et al. 1998). In those simulations, a spherical kernel was used, where collisions were not enabled. The use of a cylindrical kernel in the superparticle approach is justified because the superparticle approach treats collisions in a statistical fashion, where the interacting superparticles are considered to fill the entire grid cell.

Collisions are enabled at the same time when the simulation starts with $\mathbf{u}=0$. This yields virtually the same result compared to the case when turbulence is already fully developed and droplets are mixed (see appendix B). Since collisions can only happen when a pair of superparticles resides in the same grid cell, it is important to have sufficient statistics of initial $N_{p} / N_{\text {grid }}\left(N_{p}\right.$ is the number of superparticles and $N_{\text {grid }}$ is the number of grid cells). Furthermore, to obtain fully developed turbulence, a large number of mesh points $N_{\text {grid }}\left(512^{3}\right.$ in the present study) is essential. This requires a large number of superparticles, which is computationally expensive even using the modern supercomputers. We investigate the statistical convergence with respect to the initial value of $N_{p} / N_{\text {grid }}$ and find that it is converged at 0.05 (see appendix $C$ ). Nevertheless, to have sufficient droplet statistics, we adopt $N_{p} / N_{\text {grid }}=0.5$, so we have on average one superparticle for every two grid cells. This makes the computation affordable since the computational cost scales as $N_{p}^{2}$. Droplet growth by condensation is not incorporated in our model. We refer to $\mathrm{Li}$ et al. (2017) for a detailed description of our numerical setup and of the algorithm used to model collision.

The superparticle approach is computationally efficient (Li et al. 2017; Shima et al. 2009; Johansen et al. 2012), but it is an approximation. How accurately it describes the actual microscopic collision dynamics depends on several factors. In the limit where the number of droplets per superparticle tends to infinity, the algorithm reduces to a full mean-field description (Zsom and Dullemond 2008; Pruppacher et al. 1998). In the opposite limit, when the number of droplets per superparticle is small, the algorithm incorporates fluctuations in the collision processes that may be important in the dilute system that we consider here (Kostinski and Shaw 2005; Wilkinson 2016). Dziekan and Pawlowska (2017) compared the superparticle approach with the direct detection of collisions of point particles and concluded that the superparticle approach can accurately describe such fluctuations as long as the number of droplets is below 10 per superparticle. In our simulations, we assign two droplets per superparticle to ensure that the algorithm is sufficiently accurate.

In our simulations, we check for collisions at each time step, which enables us to get the size distribution $f(r, t)$ at time $t$ and droplet radius $r$. This distribution not only determines rain formation in clouds, but also the optical depth of the cloud (Beals et al. 2015).

\section{c. Initial conditions}

As initial condition, we adopt a lognormal dropletsize distribution (Nenes and Seinfeld 2003; Seinfeld and Pandis 2016) that is widely used in climate models and is supported by the in situ atmospheric measurements (Miles et al. 2000),

$$
f(r, 0)=\frac{n_{0}}{\sqrt{2 \pi} \sigma_{\mathrm{ini}} r} \exp \left[-\frac{\ln ^{2}\left(r / r_{\mathrm{ini}}\right)}{2 \sigma_{\mathrm{ini}}^{2}}\right] .
$$

Here $r_{\text {ini }}=10 \mu \mathrm{m}$ and $n_{0}=n(t=0)$ is the initial number density of droplets.

To speed up the computation by a factor of a hundred, we adopt $n_{0}=10^{10} \mathrm{~m}^{-3}$ instead of the typical value in the atmospheric clouds, $n_{\text {ref }} \equiv 10^{8} \mathrm{~m}^{-3}$ (cf. Li et al. 2017). We explore the convergence of $\sigma_{\text {ini }}$ for collision driven by combined turbulence and gravity. It is found that $\sigma_{\text {ini }}$ converges at 0.02 (see appendix D). However, since gravity-generated collision is very sensitive to the initial size difference, we employ monodisperse initial distribution $\left(\sigma_{\mathrm{ini}}=0\right)$ for the case of combined turbulence and gravity. For turbulence-generated collision without gravity, we employ $\sigma_{\text {ini }}=0.2$.

\section{Results and discussion}

\section{a. Collisions driven by turbulence}

Figure 1a shows the time-averaged turbulent kineticenergy spectra for different values of $\mathrm{Re}_{\lambda}$ at fixed $\bar{\varepsilon} \approx 0.04 \mathrm{~m}^{2} \mathrm{~s}^{-3}$. Here, $\mathrm{Re}_{\lambda}$ is varied by changing the domain size $L$, which in turn changes $u_{\mathrm{rms}}$. For larger Reynolds numbers the spectra extend to smaller wavenumbers. Since the energy spectrum is compensated by $\bar{\varepsilon}^{-2 / 3} k^{5 / 3}$, a flat profile corresponds to Kolmogorov scaling (Pope 2000). For the largest $\mathrm{Re}_{\lambda}$ in our simulations $\left(\operatorname{Re}_{\lambda}=158\right)$, the inertial range extends for about a decade in $k$ space. Figure $1 \mathrm{~b}$ shows how the energy spectra depend on $\bar{\varepsilon}$. Here we keep the values of $\operatorname{Re}_{\lambda}$ and $\nu$ fixed, but vary $u_{\mathrm{rms}}$ by changing both $L$ and the amplitude of the forcing (see Table 1 for details). Since the abscissa in the figures is normalized by $k_{\eta}=2 \pi / \eta$, the different spectra shown in Fig. 1b collapse onto a single curve.

Figure 2a shows the droplet-size distributions obtained in our simulations for different values of $\mathrm{Re}_{\lambda}$, but 

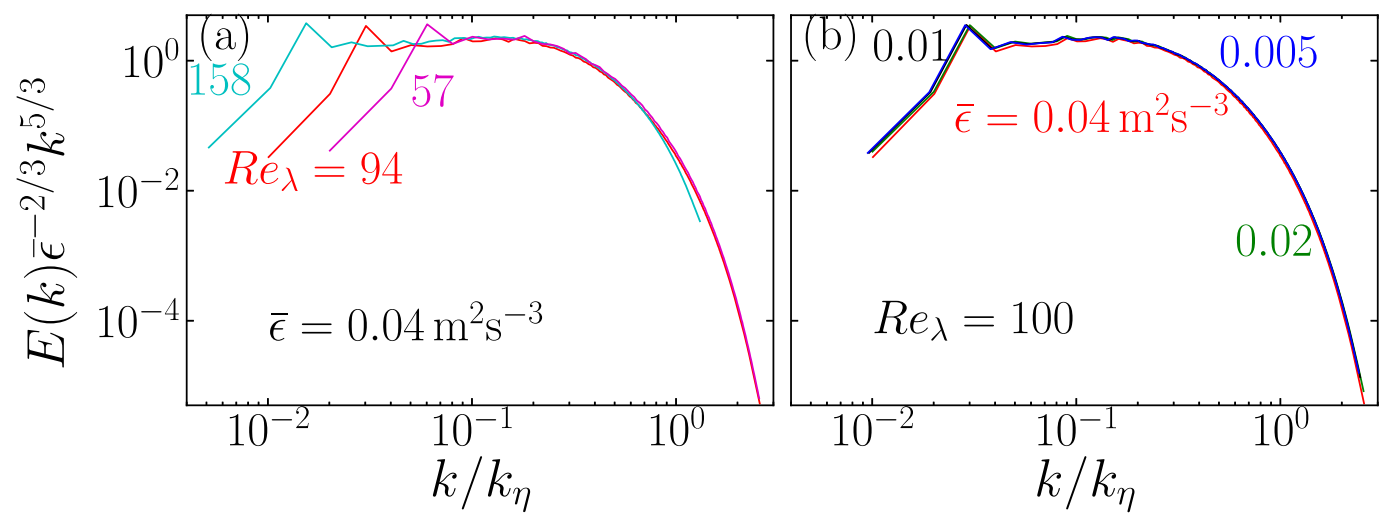

FIG. 1. Time-averaged kinetic energy spectra of the turbulence gas flow for (a) different $\operatorname{Re}_{\lambda}=57$ (magenta dashed line), 94 (red solid line), and 158 (cyan dotted line) at fixed $\bar{\varepsilon}=0.04 \mathrm{~m}^{2} \mathrm{~s}^{-3}$ (see runs A, B, and C in Table 1 for details) and for (b) different $\bar{\varepsilon}=0.005$ (blue dotted line), 0.01 (black dashed line), 0.02 (green dash-dotted line), and $0.04 \mathrm{~m}^{2} \mathrm{~s}^{-3}$ (read solid line) at fixed $\mathrm{Re}_{\lambda}=100$ (see runs B, D, E, and F in Table 1 for details).

for the same $\bar{\varepsilon}$. This figure demonstrates that the time evolution of the size distribution depends only weakly on $\operatorname{Re}_{\lambda}$ when $\bar{\varepsilon}$ is kept constant. This is consistent with the notion that the collisional growth is mainly dominated by the Kolmogorov scales (Saffman and Turner 1956; Devenish et al. 2012). The maximum Reynolds number in our DNS is $\mathrm{Re}_{\lambda} \approx 158$. This value is still two orders of magnitude smaller than the typical value in atmospheric clouds (Grabowski and Wang 2013). It cannot be ruled out that there may be a stronger Reynolds-number effect on the collisional growth at higher Reynolds numbers (Shaw 2003; Ireland et al. 2016a; Onishi and Seifert 2016). In the simulations of Onishi and Seifert (2016), where collisions are detected directly, the largest value of $\mathrm{Re}_{\lambda}$ was 333 , which is twice as large as our largest value. They showed that the turbulence enhancement factor weakly depends on $\mathrm{Re}_{\lambda}$ when the mean radius of the initial distribution is $10 \mu \mathrm{m}$. This is consistent with our results.

Figure $2 \mathrm{~b}$ shows how the evolution of the droplet-size distribution depends on $\bar{\varepsilon}$, for a fixed $\operatorname{Re}_{\lambda}$. We see that especially the tails of the size distributions depend

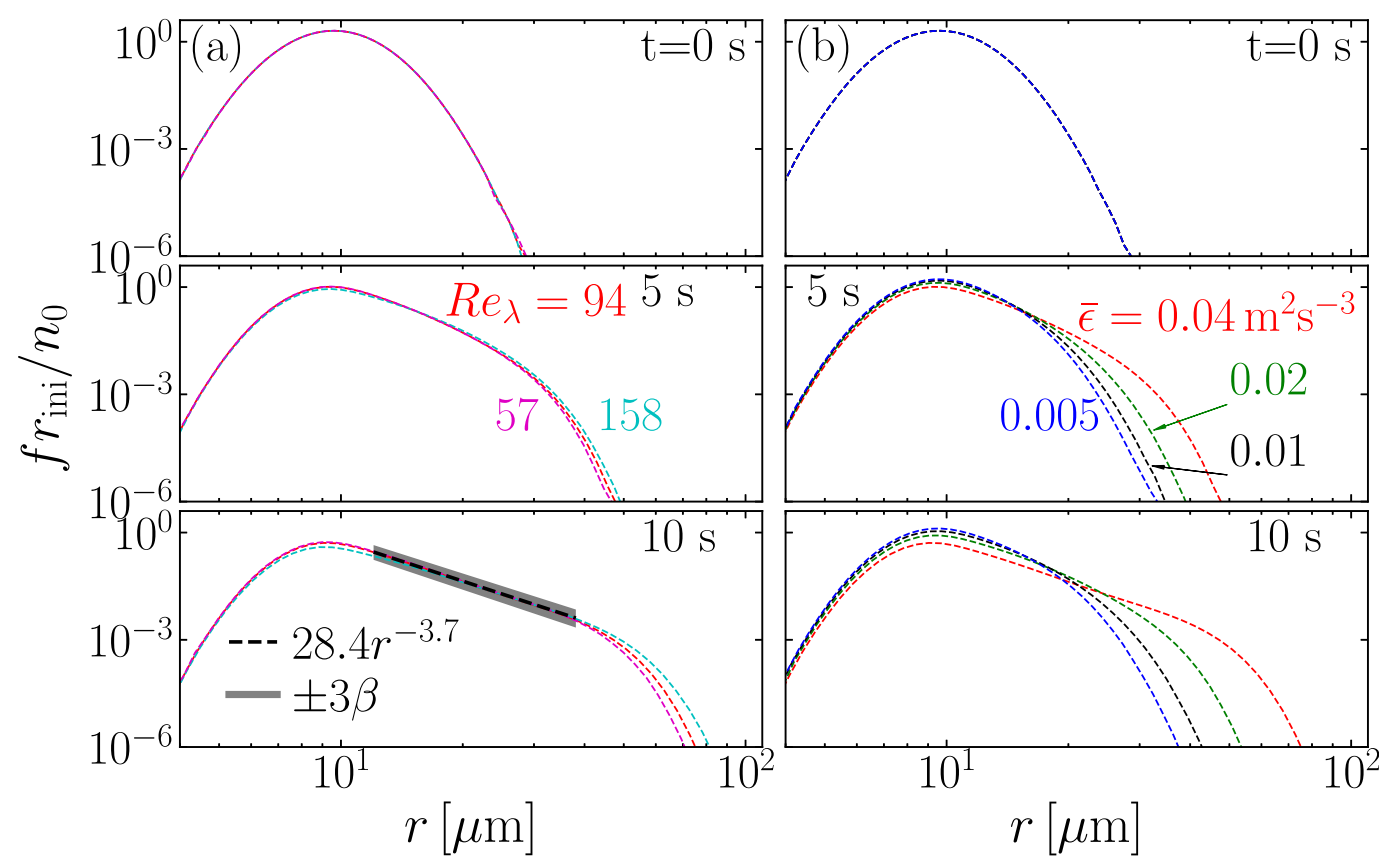

FIG. 2. Droplet size distribution for the same simulations as in Fig. 1. (a) Different $\operatorname{Re}_{\lambda}$ at fixed $\bar{\varepsilon}$. (b) Different $\bar{\varepsilon}$ at fixed $\operatorname{Re}_{\lambda}$. Here $\beta$ is the standard deviation and $3 \beta$ is the significance level. 


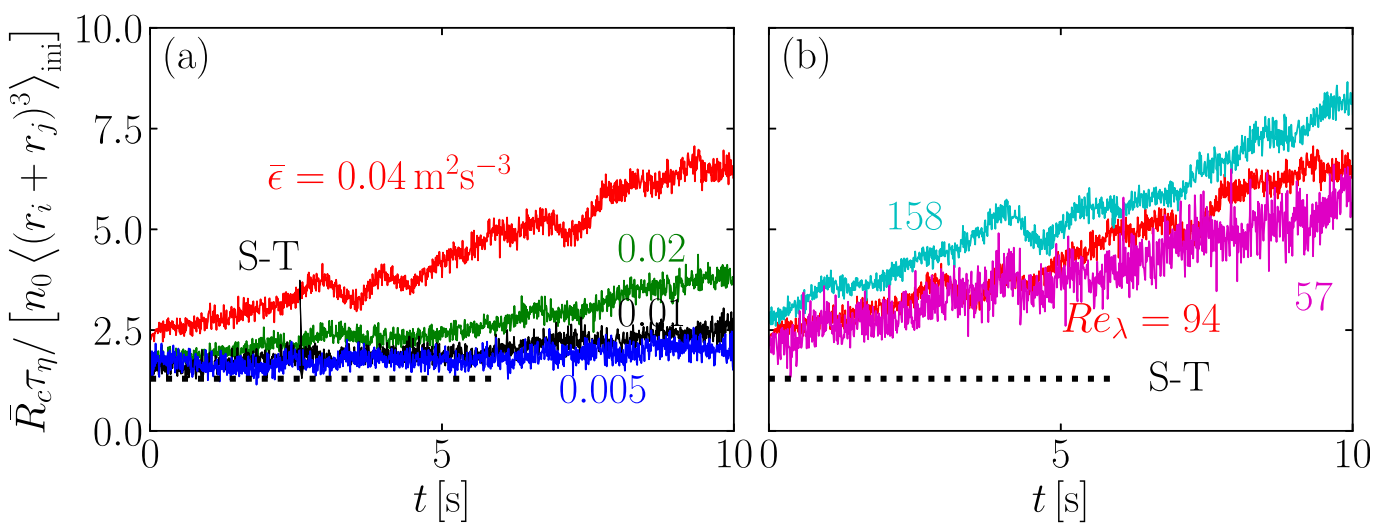

FIG. 3. Mean collision rate for same simulations as in Fig. 1. (a) Different $\bar{\varepsilon}$ at fixed $\operatorname{Re}_{\lambda}$. (b) Different $\operatorname{Re}_{\lambda}$ at fixed $\bar{\varepsilon}$. In both panels the data are normalized by dividing by $n_{0}\left\langle\left(r_{i}+r_{j}\right)^{3}\right\rangle_{\mathrm{ini}} / \tau_{\eta}$.

strongly on $\bar{\varepsilon}$ : the larger $\bar{\varepsilon}$, the wider are the tails. The tails in the droplet size distribution lead to a broad distribution of Stokes numbers. Also, since $\mathrm{St}=\tau_{i} / \tau_{\eta} \propto \bar{\varepsilon}^{1 / 2}$, the St distribution shifts to large Stokes numbers as $\bar{\varepsilon}$ increases (see appendix E).

We now show that the $\bar{\varepsilon}$ dependence of the size distribution is due to the sensitive dependence of the collision rate upon this parameter. Figure 3 shows how the mean collision rate $\bar{R}_{c}$ changes as a function of time. This rate, which depends implicitly on $\bar{\varepsilon}$, is defined as

$$
\bar{R}_{c}=\pi n_{0}(2 r)^{2} \overline{|\mathbf{v}|},
$$

where $\mathbf{v}$ is the relative velocity between two approaching droplets. This expression is written for identical droplets with radius $r$. In bidisperse suspensions with droplets of two different radii $r_{i}$ and $r_{j}, 2 r$ is replaced by $r_{i}+r_{j}$. Collisions of small droplets advected by turbulence are due to local turbulent shear, provided that droplet inertia is negligible. Saffman and Turner (1956) proposed an expression for the resulting collision rate:

$$
R_{c}^{\mathrm{S}-\mathrm{T}}=\frac{C n_{0}(2 r)^{3}}{\tau_{\eta}} .
$$

Saffman and Turner (1956) quote the value $C=$ $\sqrt{8 \pi / 15} \approx 1.29$ for the prefactor, but this is just an approximation, even at $\mathrm{St}=0$ (Voßkuhle et al. 2014). It turns out that the Saffman-Turner estimate is an upper bound (Gustavsson and Mehlig 2016a), because it counts recollisions that must not be counted when the droplets coalesce upon collision, as in our simulations. Here recollision means that one droplet can experience several collisions since there is no coalescence. DNS of small droplets in turbulence also count recollisions (no coalescence) and yield a value of $C$ in good agreement with the Saffman-Turner estimate (Voßkuhle et al. 2014), in the limit of $\mathrm{St} \rightarrow 0$.

In Fig. $3 \mathrm{a}$ we normalized the mean collision rate by dividing with the Saffman-Turner expression in Eq. (10) for the collision rate, averaging $(2 r)^{3}=\left(r_{i}+r_{j}\right)^{3}$ over the initial size distribution. In this way, we obtain the coefficient $C$ from the output of the mean collision rate $\bar{R}_{c}$. Initially, the collision rate is of the same order as predicted by Eq. (10), but in our simulations the coefficient $C$ depends on $\varepsilon$. It ranges from $C \approx 1.57$ at $\bar{\varepsilon}=0.005 \mathrm{~m}^{2} \mathrm{~s}^{-3}$ to $C \approx 2.26$ at $\bar{\varepsilon}=0.04 \mathrm{~m}^{2} \mathrm{~s}^{-3}$. All values are larger than the Saffman-Turner prediction in spite that the Saffman-Turner collision rate is argued to be an upper bound for advected droplets (Gustavsson et al. 2008a). However, in our simulations the mean Stokes number ranges from $\mathrm{St}=0.05$ for $\bar{\varepsilon}=0.005 \mathrm{~m}^{2} \mathrm{~s}^{-3}$ to $\mathrm{St}=0.14$ for $\bar{\varepsilon}=0.04 \mathrm{~m}^{2} \mathrm{~s}^{-3}$. From Fig. 1 of Voßkuhle et al. (2014) we infer that $C=1.9$ for $\mathrm{St}=0.05$, in reasonable agreement with our simulation results. However, their $C=5$ for $\mathrm{St}=0.14$, which is about twice as large as our value $(C \approx 2.26)$. This overestimation of $C$ at $\mathrm{St}=0.14$ could be due to their recollisions. We conclude that the collision rate scales initially as predicted by the Saffman-Turner theory, $R_{c} \sim \sqrt{\bar{\varepsilon}}$, with small corrections due to particle inertia. At later times these corrections become larger. In recent years, several works have indicated that the Saffman-Turner model underestimates the collision rate at larger Stokes numbers when the effect of droplet inertia becomes important, so that the droplets can detach from the flow. Model calculations show that this can substantially enhance the collision rate. Two mechanisms have been proposed.

First, droplet inertia causes identical droplets to cluster spatially (Maxey 1987; Elperin et al. 1996, 2002; 
Reade and Collins 2000; Kostinski and Shaw 2001; Bec 2003; Duncan et al. 2005; Elperin et al. 2013; Gustavsson and Mehlig 2016b). At small spatial scales the clustering of identical droplets is fractal. This enhances the collision rate of small droplets (Gustavsson et al. 2008a): $R_{c}=C n_{0}(2 r)^{3} \tau_{\eta}^{-1} g(2 r)$. Here $g(2 r)$ is the pair correlation function measuring the degree of fractal clustering of identical droplets: $g(2 r)$ diverges $\sim r^{-\xi}$ as $r \rightarrow 0$ with $\xi>0$. The exponent $\xi$ has been computed in DNS and model calculations (Gustavsson and Mehlig 2016b). It has a weak dependence on $\bar{\varepsilon}$. However, $g(2 r)$ is calculated based on the particle field with a single Stokes number, which makes it impossible to attempt a quantitative comparison between this theory and our simulation data. More importantly, collision leads to a distribution of droplet sizes. Droplets of different sizes cluster onto different fractal attractors. This may reduce the effect of spatial clustering on the collision rate (Chun et al. 2005b; Bec et al. 2005; Meibohm et al. 2017).

Second, singularities in the droplet dynamics (caustics) give rise to multivalued droplet velocities, resulting in large velocity differences between nearby droplets (Sundaram and Collins 1997a; Falkovich et al. 2002; Wilkinson et al. 2006; Falkovich and Pumir 2007; Gustavsson and Mehlig 2014; Voßkuhle et al. 2014). Most model calculations were performed for identical droplets. They indicate that the enhancement of the collision rate due to multivalued droplet velocities dominates for Stokes numbers larger than unity (Voßkuhle et al. 2014). In this case, a Kolmogorovscaling argument suggests (Mehlig et al. 2007; Gustavsson et al. 2008b) that $R_{c} \sim n_{0} r^{2} u_{K} \sqrt{\mathrm{St}} \propto \bar{\varepsilon}^{1 / 2}$, where $u_{K}$ is the turbulent velocity at the Kolmogorov scale; this $\sqrt{\mathrm{St}}$ dependence was first suggested by Völk et al. (1980) using a different argument. This expression has the same $\bar{\varepsilon}$ dependence as Eq. (10). We note, however, that the Kolmogorov-scaling argument leading to this $\sqrt{\mathrm{St}}$ dependence rests on the assumption that there is a welldeveloped inertial range $\left(\operatorname{Re}_{\lambda} \rightarrow \infty\right)$. This assumption is not fulfilled in our simulations. Moreover, at later times we expect that collisions between droplets of different sizes make an important contribution (Meibohm et al. 2017). Scaling theory (Mehlig et al. 2007) suggests that the $\bar{\varepsilon}$ scaling remains the same in the limit of $\operatorname{Re}_{\lambda} \rightarrow \infty$. But, again, this limit is not realized in our simulations. Also, any theory for the collision rate in bidisperse suspensions must be averaged over the distribution of particle sizes and their velocities to allow comparison with Fig. 3a. This may introduce additional $\bar{\varepsilon}$ dependencies. It is therefore plausible that the small-St scaling, $R_{c} \sim \sqrt{\bar{\varepsilon}}$, breaks down in our simulations at larger Stokes numbers, indicating that the increase in the mean collision rate could be an inertial effect. Moreover, since the Stokes numbers are larger for larger values of $\bar{\varepsilon}$, we expect the inertial additive corrections of the collision rate (due to clustering and increased relative particle velocities) to be larger at larger $\bar{\varepsilon}$. This is consistent with Fig. 3a. In conclusion, the mean collision rate depends strongly on $\bar{\varepsilon}$ (Fig. 3a), as do the size distributions shown in Fig. $2 b$.

Figure $3 \mathrm{~b}$ shows that the mean collision rate depends only weakly on the Reynolds number. It demonstrates that the collision rate is somewhat larger for larger Reynolds numbers. This is consistent with the notion that particle pairs exploring the inertial range collide at larger relative velocities when the inertial range is larger (Gustavsson et al. 2008b). But, as pointed out above, the inertial range in our simulations is too small for this mechanism to have a substantial effect.

It is interesting to note that the size distribution exhibits power-law behavior in the range of $10-40 \mu \mathrm{m}$, as shown in the third panel of Fig. 2a. A slope of -3.7 is observed. Remarkably, similar power laws have been observed in several other circumstances, where the collisional growth is not subjected to gravity. First, the observed size distribution of interstellar dust grains shows a power law with a slope of $-3.3, \ldots,-3.6$ (Mathis et al. 1977). The collisional growth of such dust grains in a turbulent environment is one of the main mechanisms for planet formation (Johansen and Lambrechts 2017). Another example concerns the size distribution of particles in Saturn's rings (Brilliantov et al. 2015), where a slope of -3 is observed. This power-law size distribution may be universal for turbulencegenerated collisional growth. Therefore, turbulencegenerated collisional growth without or with weak gravity is relevant to other applications. Next, to understand the warm rain formation, we compare with the case where gravity is included.

\section{b. Collisions driven by combined turbulence and gravity}

For cloud droplet growth, gravitational settling is significant (Woittiez et al. 2009; Grabowski and Wang 2013). Collision driven by gravity is very sensitive to the initial size difference (see appendix D). To avoid any bias from the initial size difference, we adopt a monodisperse initial distribution, that is, $\sigma_{\text {ini }}=0$. In Fig. 4 , we compare the evolution of the droplet-size distribution for the turbulent case and the combined case with turbulence and gravity. At $t=1 \mathrm{~s}$, both cases have almost the same droplet-size distribution, demonstrating that turbulence dominates the collisional growth. When $t \geq 1 \mathrm{~s}$, gravity dominates the time evolution of the droplet-size distribution. The tail of the dropletsize distribution reaches $80 \mu \mathrm{m}$ (drizzle size) for the 


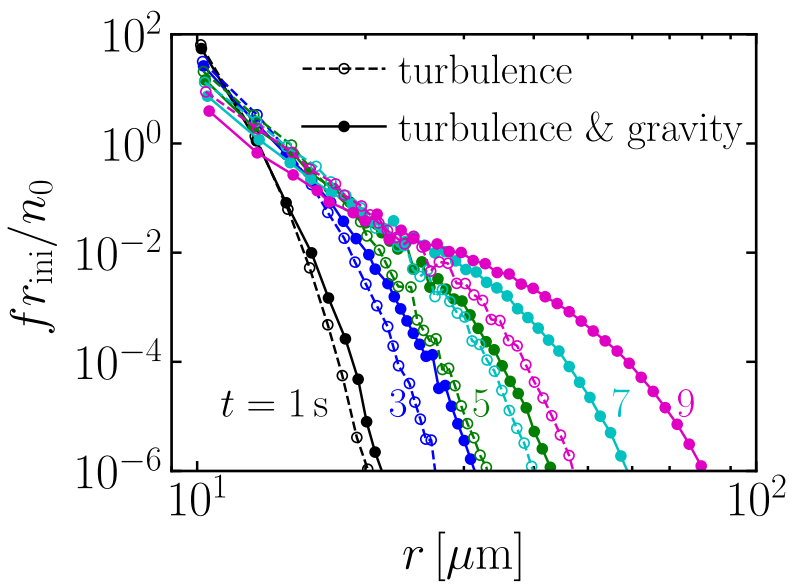

FIG. 4. Time evolution of the droplet size distribution. Comparing the pure turbulence case (open symbols) with the turbulence and gravity case (filled symbols). The time interval is $2 \mathrm{~s}$, plotted from 1 to $9 \mathrm{~s}$ (from left to right). The mean energy dissipation rate is $\bar{\varepsilon}=0.04 \mathrm{~m}^{2} \mathrm{~s}^{-3}$ and $\mathrm{Re}_{\lambda}=100$. The droplets are all of size $10 \mu \mathrm{m}$ initially; see run $\mathrm{D}$ in Table 1 for details of the simulation.

combined turbulence and gravity case at $t=9 \mathrm{~s}$. For the turbulence case, the tail reaches $48 \mu \mathrm{m}$ after the same time, which is roughly half the radius obtained for the combined turbulence and gravity case. Since our initial number density of cloud droplets is 100 times larger than the typical value in atmospheric clouds, we can scale our simulation time by a factor of 100 . Thus, a scaled time of $9 \mathrm{~s}$, for example, corresponds to $900 \mathrm{~s}$ in atmospheric clouds. This rescaling is validated in appendix $F$, where the tail of the size distribution is found to differ by only $5 \mu \mathrm{m}$ in radius for $n_{0}=10^{10}$ and $n_{0}=10^{8} \mathrm{~m}^{-3}$. We find that collisional growth of cloud droplets can reach drizzle-sized droplets in about $900 \mathrm{~s}=15 \mathrm{~min}$. This is comparable to the time scale for rapid warm rain formation. This time scale, however, is expected to change if a turbulence-induced collision efficiency were to be taken into account. Besides, the cloud system is about 100 times more dilute in particle number density, which may also change the time scale.

Next, we check the $\bar{\varepsilon}$ dependency for the combined turbulence and gravity case. As shown in Fig. 5, the tail of the size distribution broadens with increasing $\bar{\varepsilon}$. When $f r_{\text {ini }} / n_{0}=10^{-6}$ at $t=10 \mathrm{~s}$, the radius resulting from $\bar{\varepsilon}=0.04 \mathrm{~m}^{2} \mathrm{~s}^{-3}$ is about $60 \mu \mathrm{m}$, while the one resulting from $\bar{\varepsilon}=0.005 \mathrm{~m}^{2} \mathrm{~s}^{-3}$ is about $90 \mu \mathrm{m}$. The $30-\mu \mathrm{m}$ difference means turbulence efficiently enhance the collisional growth when $\sigma_{\text {ini }}=0$. To quantify the role of turbulence at different phases during the collisional growth, we inspect the mass distribution function (Berry and Reinhardt 1974). We use the same nomenclature of the mass distribution as Berry and Reinhardt

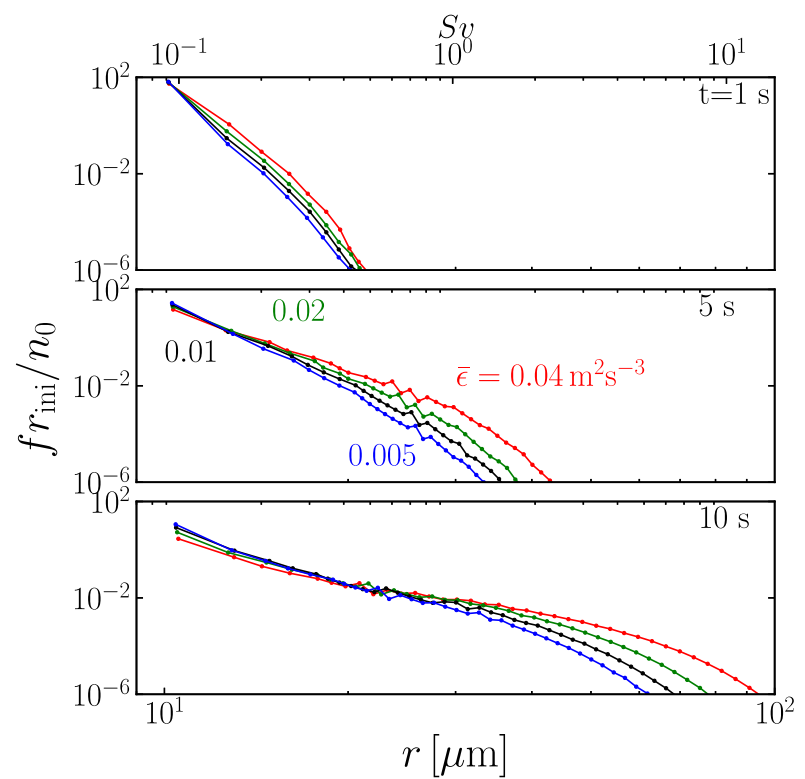

FIG. 5. Time evolution of the droplet size distribution for different $\bar{\varepsilon}$ in combined turbulence and gravity environment and with $\operatorname{Re}_{\lambda}=100$. Droplets are all with size $10 \mu \mathrm{m}$ initially. See runs A-D in Table 1 for details of the simulations.

(1974), $g(\ln r, t)$. The mean mass of liquid water in terms of the size distribution function $f(r, t)$ is $\bar{M}=$ (4/3) $\pi \rho_{d} \int_{0}^{\infty} f(r, t) d r$, which is $\bar{M}=\int_{0}^{\infty} g(\ln r, t) d \ln r$ in terms of $g(\ln r, t)$. Therefore, $g(\ln r, t)=(4 \pi / 3) \rho_{d} r^{4} f(r, t)$. Figure 6 shows $g(\ln r, t)$ calculated from the same simulations as in Fig. 5, where the collision is driven by both gravity and turbulence $\left(\sigma_{\mathrm{ini}}=0\right)$. At $t=1 \mathrm{~s}$, turbulence enhances the autoconversion phase as shown by the first peaks, when $10-\mu \mathrm{m}$-sized droplets collide. The enhancement factor (amplitude of the peaks) scales almost linearly with $\bar{\varepsilon}$. This enhancement at the autoconversion phase leads to faster growth of droplet with increasing $\bar{\varepsilon}$ at late times (i.e., $t=5$ and $10 \mathrm{~s}$.). Therefore, we see a faster growth of large droplets with increasing $\bar{\varepsilon}$ at late times virtually. This is also consistent with the conclusion from Fig. 4 that turbulence dominates the collisional growth at the early stage of cloud droplets formation. Additionally, this also implies that the turbulence enhancement effect is the most efficient when the size of a colliding pairs is comparable, which is consistent with previous findings (Pinsky et al. 2007; Ayala et al. 2008a; Chen et al. 2018).

When gravity is included, the nondimensional terminal velocity $\mathrm{Sv}=v_{g} / u_{\eta}$, charactering the relative droplet inertia and gravitational sedimentation, becomes important (Devenish et al. 2012) [here we adopt Sv because it contains the information of particle size compared with the Froude number of particles defined as $\mathrm{Fr}=|\mathbf{g}| \tau_{\eta} / u_{\eta}$ (Gustavsson et al. 2014), and Sv can 


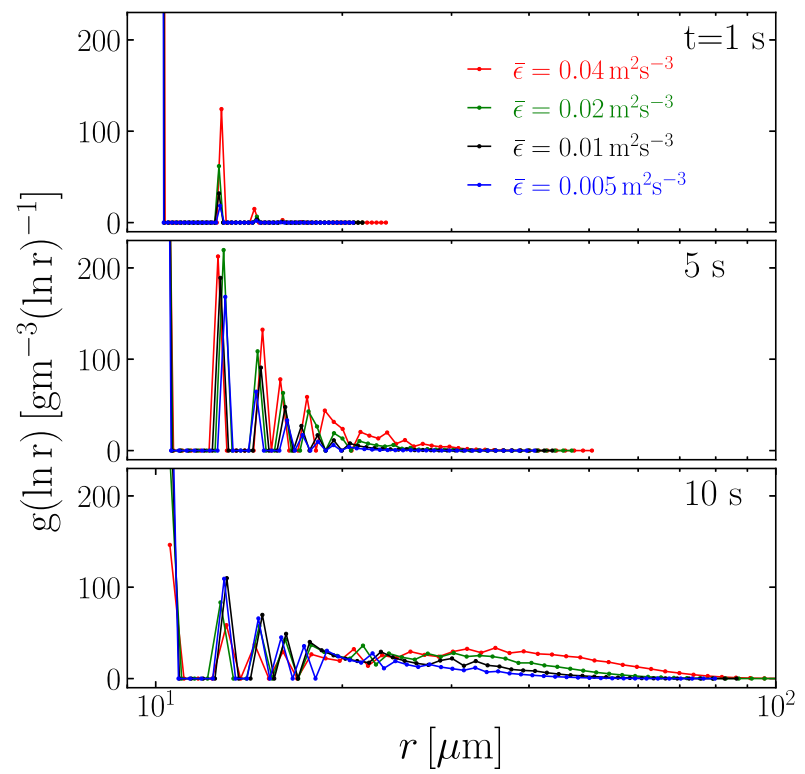

FIG. 6. Time evolution of the mass distribution function $g(\ln r, t)$. Same simulations as in Fig. 5. The values of the peaks at $t=1 \mathrm{~s}$ are $18.39\left(\bar{\varepsilon}=0.005 \mathrm{~m}^{2} \mathrm{~s}^{-3}\right), 31.92\left(\bar{\varepsilon}=0.01 \mathrm{~m}^{2} \mathrm{~s}^{-3}\right), 61.83(\bar{\varepsilon}=$ $\left.0.005 \mathrm{~m}^{2} \mathrm{~s}^{-3}\right)$, and $124.26\left(\bar{\varepsilon}=0.005 \mathrm{~m}^{2} \mathrm{~s}^{-3}\right)$.

be expressed as $\mathrm{Sv}=\mathrm{FrSt}]$, where $v_{g}=\tau_{i}|\mathbf{g}|$ is the terminal fall velocity and $u_{\eta}$ is the turbulent velocity at the Kolmogorov scale $\eta$. It can also be interpreted as the ratio of the Kolmogorov eddy turnover time and the time it takes for a particle to sediment across the eddy. If the ratio is much larger than unity, the particle will rapidly sediment through the eddy, thereby leading to weak particle-eddy interaction. On the other hand, if $\mathrm{Sv}$ is much smaller than unity, sedimentation does not play a significant role in reducing the time of particleeddy interaction (Ayala et al. 2008b). The distribution of Sv shows the same dependency on $\bar{\varepsilon}$ as $f(r, t)$ in our simulations when $\sigma_{\text {ini }}=0$, as demonstrated in Fig. 5.

Further inspection of the mean collision rate $\bar{R}_{c}$ (Fig. 7) is consistent with the above observations. More importantly, the normalized $\bar{R}_{c}$ collapse onto each other and follow exponential growth. The collapse reconciles our finding that the turbulence enhancement at the autoconversion phase scales with $\bar{\varepsilon}$. The exponential growth of $\bar{R}_{c}$ can be explained by the following theory of the continuous collision (Lamb and Verlinde 2011). Given two droplets of very different sizes that collide with each other due to gravity, the collision rate given by Eq. (7) is

$$
\bar{R}_{c}^{\text {continuous }}=\pi\left(r_{L}+r_{S}\right)^{2}\left|\mathbf{V}_{L}-\mathbf{V}_{S}\right| .
$$

Substituting Eq. (6) into Eq. (5), and taking into account that $\mathrm{Re}_{i} \sim V_{i} r_{i}$, we obtain $\tau_{i} \sim r_{i}^{2} /\left(V_{i} r_{i}\right)^{2 / 3}=r_{i}^{4 / 3} V_{i}^{-2 / 3}$.

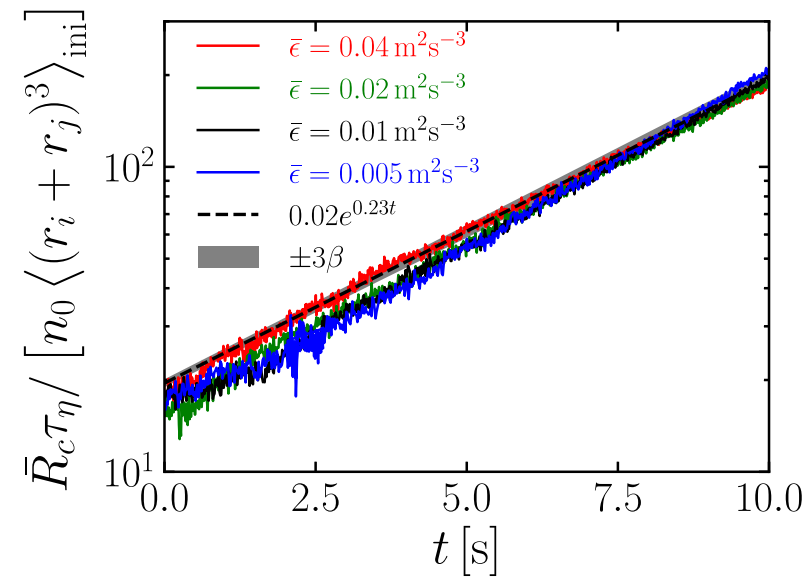

FIG. 7. Mean collision rate $\bar{R}_{c}$ for different $\bar{\varepsilon}$. Same simulations as in Fig. 5. Here $\beta$ is the standard deviation and $3 \beta$ is the significance level.

When gravity dominates the motion of a droplet, the droplet velocity is of the order of the terminal fall velocity, $V_{i}=\tau_{i} g$, so that $V_{i} \sim r_{i}^{4 / 5}$. The linear approximation for the velocity (Lamb and Verlinde 2011) is now obtained by replacing the exponent $4 / 5$ with unity, such that Eq. (11) simplifies to

$$
\bar{R}_{c}^{\text {continuous }} \sim r_{L}^{3}
$$

when $r_{L} \gg r_{S}$. The rate of mass increase, $d m_{L} / d t$, is proportional to the collision rate. Therefore, Eq. (12) can also be expressed as

$$
\frac{d m_{L}}{d t} \sim r_{L}^{3}
$$

Combining Eqs. (12) and (13), we can obtain the exponential growth of $\bar{R}_{c}$,

$$
\bar{R}_{c}^{\text {continuous }} \sim \exp (\alpha t),
$$

where $\alpha$ is a constant.

The excellent agreement of $\bar{R}_{c}$ between our simulation and the theory demonstrates that the continuous growth theory is robust in representing the mean collision rate. Even in the circumstance that we detect the collision rate directly by counting each collision event without any assumptions, such as that of large size differences, the linear approximation for the velocity (Lamb and Verlinde 2011), and the absence of turbulence. When $\bar{R}_{c}$ is normalized by $\tau_{\eta}$, the curves representing different $\bar{\varepsilon}$ collapse onto each other (see Fig. 7). This indicates that 1) gravity dominates the collisional growth, 2) collision time scale is smaller than the Kolmogorov time scale, 3) turbulence is responsible for generating few larger droplets so that the gravitational 

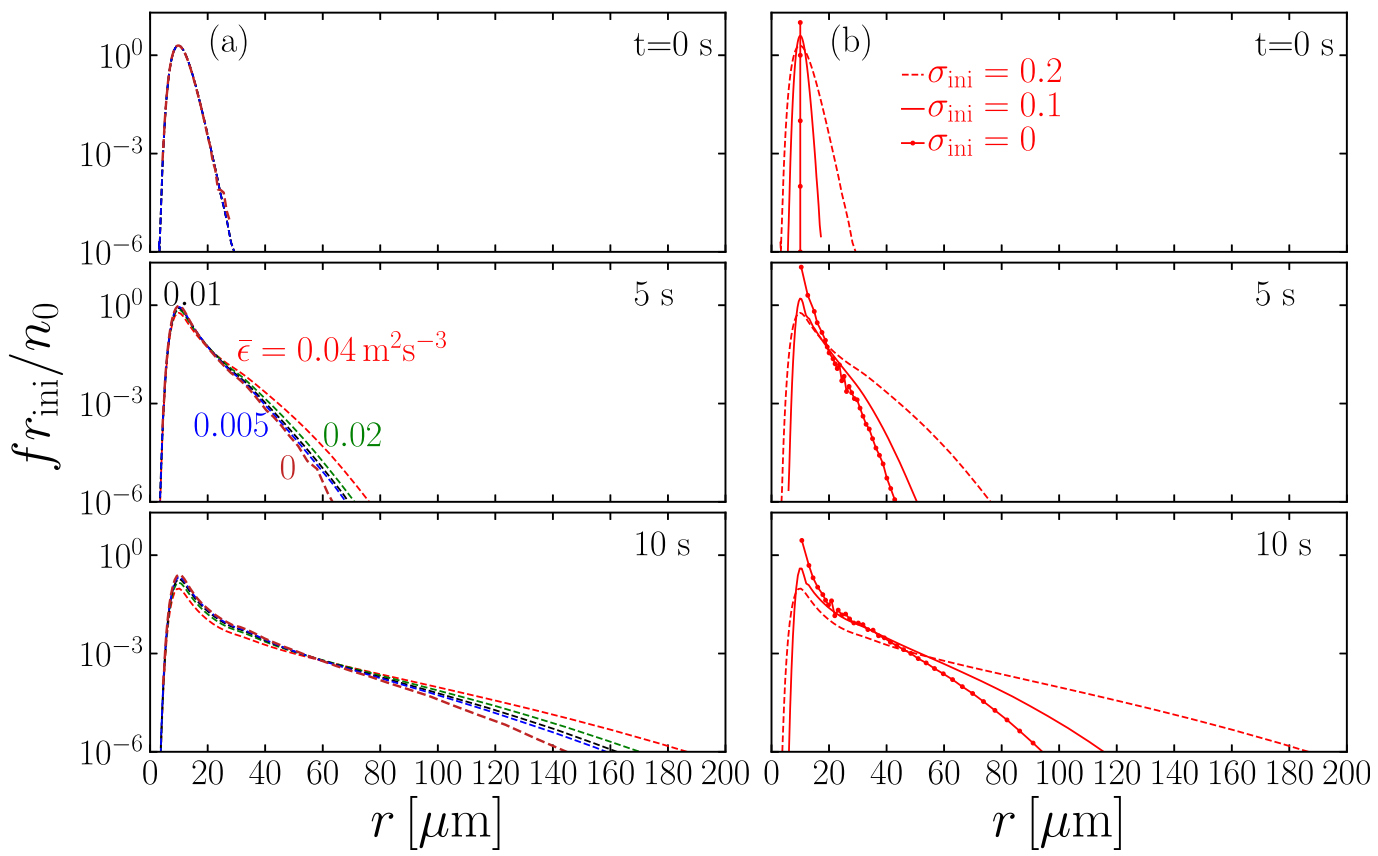

FIG. 8. Same simulations as in Fig. 2, but with gravity invoked. (a) Time evolution of size distribution for different $\bar{\varepsilon}$ with $\sigma_{\text {ini }}=0.2$; the brown dashed lines represent the size distribution due to gravity-generated collision. (b) Comparison among $\sigma_{\text {ini }}=0,0.1$, and 0.2 with $\bar{\varepsilon}=0.04 \mathrm{~m}^{2} \mathrm{~s}^{-3}$.

collision can be triggered at the initial phase of raindrop formation, and 4) turbulence transport provides a mean effect for collision as implicitly indicated in Eq. (10).

The mean collision rate $\bar{R}_{c}$ is an averaged description, which ignores fluctuations. The agreement between DNS results and the theory of continuous collisions only suggests the mean effect of turbulence on collisional growth. Collisional growth due to random fluctuations in a dilute system (such as cloud system) was proposed already by Telford (1955). Kostinski and Shaw (2005) further argued that Poisson fluctuations of the collision times of settling droplets leads to a broad distribution of growth times that could potentially explain the rapid onset of rain formation. This question is further discussed by Wilkinson (2016). Therefore, quantifying the role of fluctuations in the collision process by means of analyzing the DNS data in the framework proposed by Kostinski and Shaw (2005) and Wilkinson (2016) is desired. Also, since our system is 100 times denser than the Earth's atmospheric cloud system, it is interesting to investigate how the diluteness affects the role of fluctuations on collisional growth.

In the atmospheric clouds, the size distribution of cloud droplets has a certain width. To investigate the collisional growth with a lognormal initial distribution when there is both turbulence and gravity, we use the same setup as in Fig. 2b, but with gravity included. As shown in Fig. 8a, the evolution of the droplet size distribution depends only weakly on the energy dissipation rate. This again confirms the notion that gravitygenerated collision is more sensitive to the initial size difference than the turbulence-generated collisions (see the $\sigma_{\text {ini }}$ dependency of the size distribution for turbulencegenerated collision in appendix D). To further illustrate this, we plot the time evolution of the size distribution with different initial widths (see Fig. 8b). We also compare the present numerical simulations with the idealized gravity-driven collision. Figure 8 a shows that the tail of the size distribution becomes broadening as $\bar{\varepsilon}$ increases. For the case of $\bar{\varepsilon}=0 \mathrm{~m}^{2} \mathrm{~s}^{-3}$, the tail reaches at about $142 \mu \mathrm{m}$ at $t=10 \mathrm{~s}$, while for the case of $\bar{\varepsilon}=$ $0.04 \mathrm{~m}^{2} \mathrm{~s}^{-3}$ the tail reaches at about $182 \mu \mathrm{m}$, resulting in an increase percentage of $28 \%$. Chen et al. (2018) found that the increasing percentage of the tail is about $(45-30) / 30=50 \%$ at $t=6.5 \mathrm{~min}(390 \mathrm{~s})$. Our result reveals an increasing percentage of $(75-62) / 62 \approx 21 \%$ at $t=5 \mathrm{~s}$ (equivalent to $500 \mathrm{~s}$ considering that $n_{0}=$ $10^{10} \mathrm{~m}^{-3}$ is used in our simulations). Since our initial size distribution and treatment of the collision efficiency are different from the ones of Chen et al. (2018), we cannot compare our results with theirs quantitatively. Nevertheless, our findings are consistent with the result of Chen et al. (2018) that turbulence enhances the collisional growth of cloud droplets. We recall that when $\sigma_{\text {ini }}=0$, strong dependency of $f(r, t)$ on $\bar{\varepsilon}$ is observed (see Fig. 5). This implies that the enhancement 

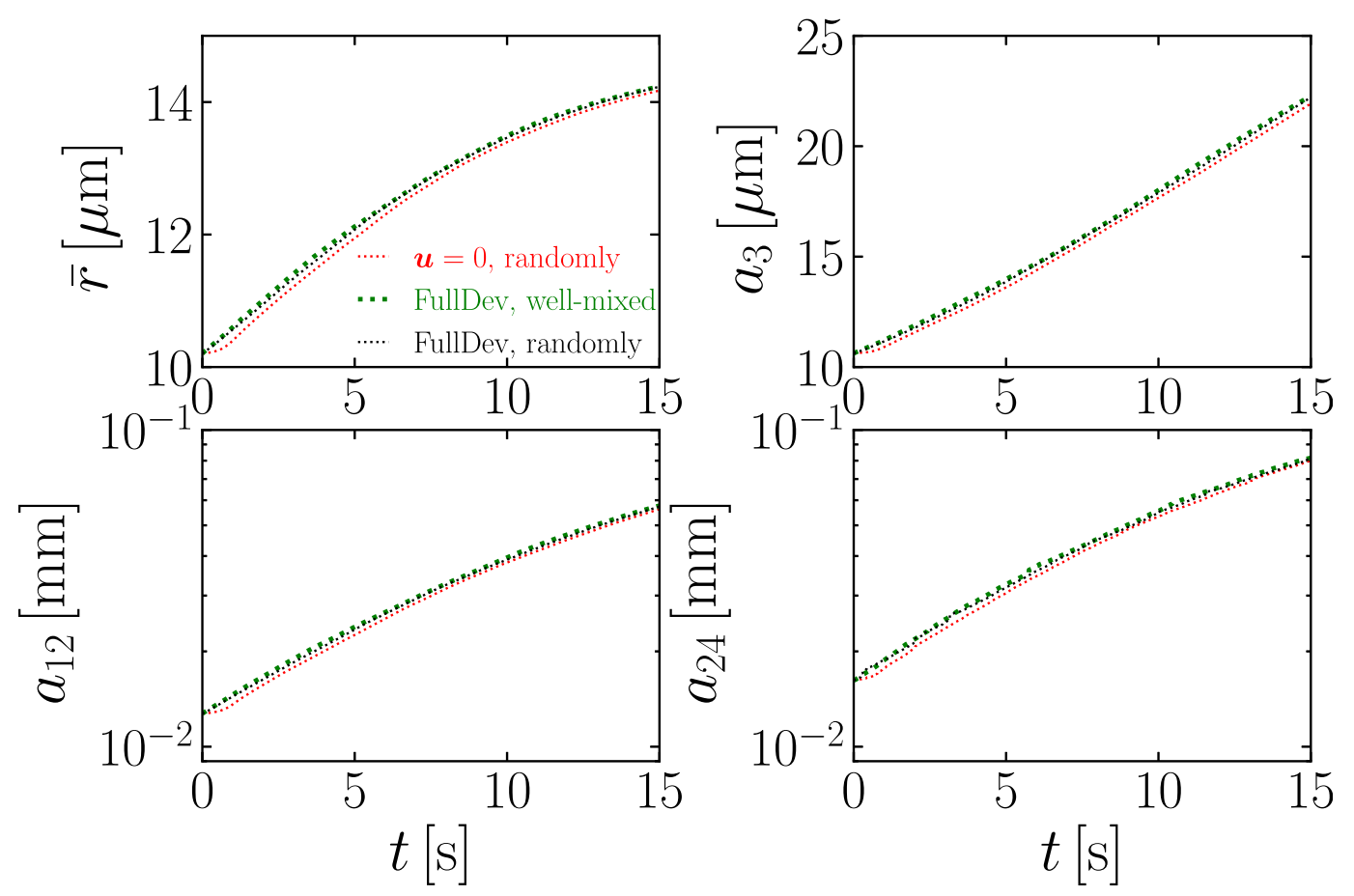

FIG. B1. Comparison of $a_{\zeta}$ for different initial conditions: collision is triggered in a randomly distributed superparticle field and the velocity of the flow is zero (red curve), in a well-mixed particle field and the velocity of the flow is zero(black curve), and in a well-mixed particle field and the turbulence is well-developed (green curve). Gravity is omitted here, and $L=0.25 \mathrm{~m}$. The initial size distribution is given by Eq. (8) with $r_{\text {ini }}=10 \mu \mathrm{m}$ and $\sigma_{\text {ini }}=0.2$. The number of mesh grid points is $128^{3}$. Parameter $f_{0}=0.02$ and $N_{p} / 10^{6}=8.4$. These result in $\operatorname{Re}_{\lambda}=100$ and $\bar{\varepsilon}=0.03 \mathrm{~m}^{2} \mathrm{~s}^{-3}$.

effect of turbulence depends on the initial distribution of cloud droplets.

\section{Conclusions}

In the present study, we have addressed the problem of turbulence effects on collisional growth of particles such as cloud droplets in the case where coalescence is included. We have investigated this effect using a superparticle approximation for the droplet dynamics in combination with high-resolution DNS of fully developed turbulence. The superparticle approach is about 10 times faster than the direct Lagrangian-detected collision method at least. In the absence of gravity, we find that the droplet size distribution depends sensitively on the mean energy dissipation rate $\bar{\varepsilon}$ at fixed $\mathrm{Re}_{\lambda}$, which we have related to the $\bar{\varepsilon}$ dependence of the mean collision rate. We find that this rate increases as $\bar{\varepsilon}^{1 / 2}$ (except for the largest values of $\bar{\varepsilon}$ simulated). This is consistent with the Saffman-Turner collision model and its extensions. A more detailed comparison with these calculations is not possible at this point, because there is no prediction for the prefactors in general. The size distribution due to turbulence-generated collisions exhibits power-law behavior with a slope of -3.7 in the size range $10, \ldots, 40 \mu \mathrm{m}$, which is close to the power-law size distribution of interstellar dust grains. This indicates that the power-law size distribution may be universal (Mathis et al. 1977). When gravity is invoked, the turbulence enhancement effect depends on the width of the initial distribution $\sigma_{\text {ini. }}$. The enhancement is the strongest when $\sigma_{\text {ini }}=0$ and weak when $\sigma_{\text {ini }}=0.2$. For the case of $\sigma_{\text {ini }}=0$, turbulence has an efficient effect at the autoconversion phase, which results in faster growth at the late stage. In atmospheric clouds, the distribution of cloud droplets always has a certain width. The role of turbulence for collisional growth should be handled with caution. To our knowledge, it is the first time that such detailed comparison between cases with or without gravity is investigated when coalescence is invoked.

When collisions are driven by both turbulence and gravity, we found that turbulence is crucial for driving the collision so that a few large cloud droplets can be formed in the initial stage of raindrop formation. Gravity takes over as the main driver for droplet collisions when the radius of cloud droplets reaches the size of about $20 \mu \mathrm{m}$. With combined turbulence and gravity, the time scale for reaching drizzle-sized droplets is about $900 \mathrm{~s}$, which is close to the time scale of the rapid warm rain formation. This time scale, however, is 

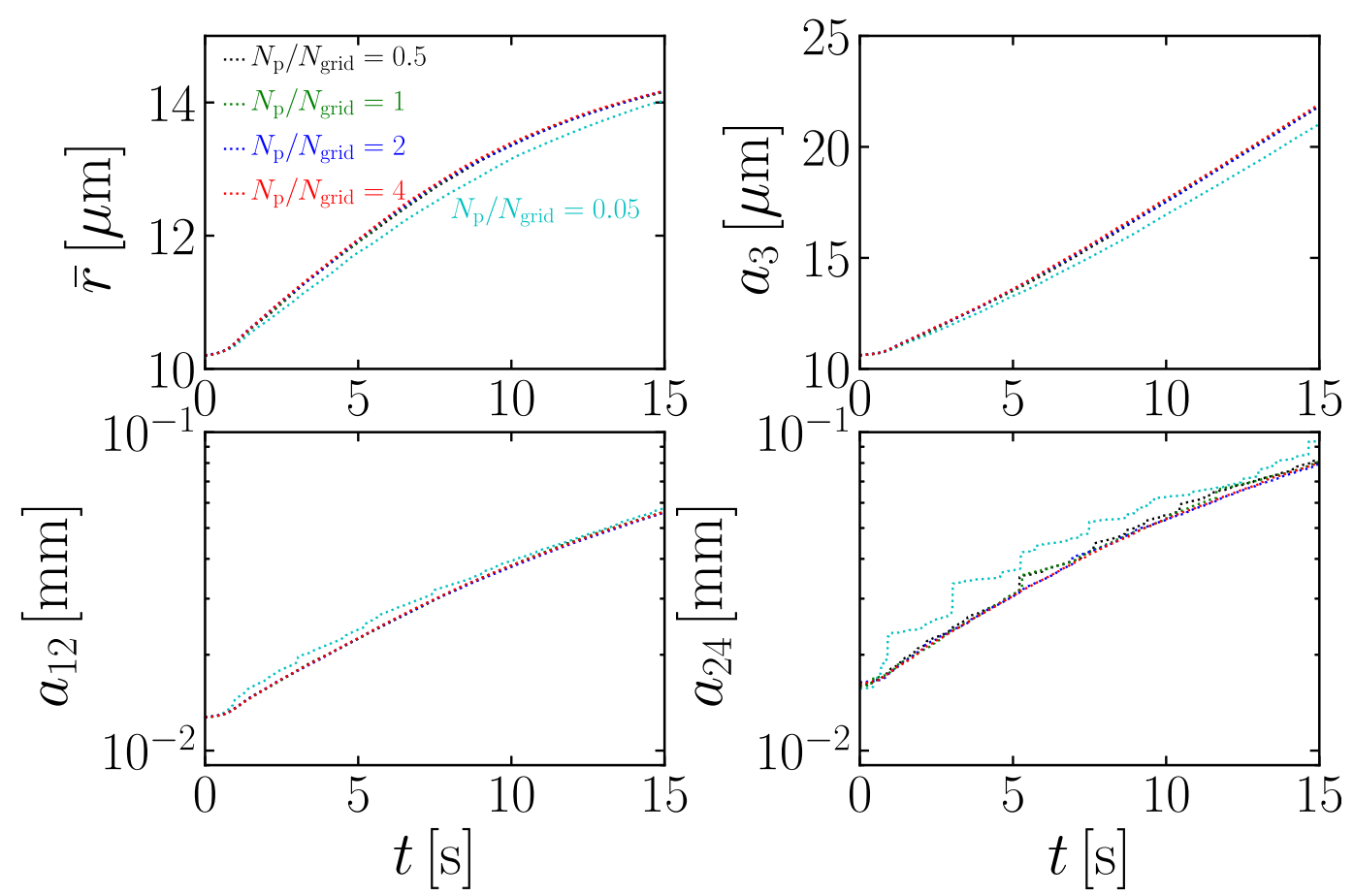

FIG. C1. Comparison of $a_{\zeta}$ for different $N_{p} / N_{\text {grid }}$. Here $N_{\text {grid }}=128^{3}$ is fixed, and $N_{p} / N_{\text {grid }}$ even converges at 0.05 . Same simulations as in Fig. 9, but with different $N_{p}$.

expected to be substantially changed when turbulenceinduced collision efficiency is taken into account. The mean collision rate grows exponentially, which is consistent with the theoretical prediction of the continuous growth even when turbulence is invoked. The theory of continuous collisions is built upon the assumptions of large size differences, a linear drag force, and gravitydriven collisions. The consistency between our simulations and the theory suggests that the theory is robust in representing the mean effect of turbulence.

The role of fluctuations for collisional growth (Telford 1955; Kostinski and Shaw 2005; Wilkinson 2016) is not explicitly analyzed. Therefore, it is interesting to investigate how the diluteness affects the role of fluctuations on collisional growth. These will be presented in a separate paper.

Collisional growth of cloud droplets due to turbulence and gravity is very sensitive to the tail of the initial size distribution. As already discussed previously ( $\mathrm{Li}$ et al. 2017), this problem is being alleviated by considering the combined condensational and collisional growth. Especially the condensational growth due to supersaturation fluctuations may result in larger tails of the size distribution (Sardina et al. 2015; Chandrakar et al. 2016). This is another subject of an ongoing separate study.

In the present paper, the collision efficiency is assumed to be unity for simplicity. In reality, the collision efficiency is not unity, but it can depend on the droplet-droplet aerohydrodynamics (Wang et al. 2005, 2007; Wang and Grabowski 2009; Chen et al. 2018). Using a unit collision efficiency overestimates the collision rate. It would be useful to incorporate the collision efficiency in turbulence invoking droplet-droplet aerohydrodynamics, but this has not yet been done. This will need to be investigated in a separate study.

Acknowledgments. We thank Akshay Bhatnagar, Gregory Falkovich, and Vladimir Zhdankin for stimulating discussions. We also thank the three anonymous reviewers for their constructive suggestions and efforts to help improving our manuscript. This work was supported through the FRINATEK Grant 231444 under the Research Council of Norway, SeRC, the Swedish Research Council Grants 2012-5797, 2013-03992, and 2017-03865, the Fromas Grant 2014-585, the Israel Science Foundation governed by the Israel Academy of Sciences Grant 1210/15 (Igor Rogachevskii), the University of Colorado through its support of the George Ellery Hale visiting faculty appointment, and the grant "Bottlenecks for particle growth in turbulent aerosols" from the Knut and Alice Wallenberg Foundation, Dnr. KAW 2014.0048. Gunilla Svensson also thanks the Wenner-Gren Foundation for their support. The simulations were performed using resources provided by the Swedish National Infrastructure for Computing (SNIC) at the Royal Institute of Technology in Stockholm and 
Chalmers Centre for Computational Science and Engineering (C3SE). This work also benefited from computer resources made available through the Norwegian NOTUR program, under Award NN9405K (Nils Haugen). The source code used for the simulations of this study, the Pencil Code, is freely available on https:/github. com/pencil-code/. The input files as well as some of the output files of the simulations listed in Table 1 are available under http://www.nordita.org/ brandenb/ projects/collision_turbulence/.

\section{APPENDIX A}

\section{Collision Algorithm of the Superparticle Scheme}

A detailed study of the superparticle approach is given in $\mathrm{Li}$ et al. (2017), where its evaluation and advantages over the Eulerian approach are investigated. Here, we briefly review the collision scheme used in the present study. When two superparticles $i$ and $j$ residing in the same grid cell collide with each other, the new masses of the particles in the two superparticles after collision obey mass conservation and are given by

$$
\begin{aligned}
& \tilde{m}_{i}=m_{i}+m_{j}, \\
& \tilde{m}_{j}=m_{j} .
\end{aligned}
$$

We assume $n_{j}>n_{i}$ without loss of generality, where $n_{j}$ and $n_{i}$ are the number density of droplets in superparticles $i$ and $j$, respectively. Their new particle number densities are

$$
\begin{aligned}
& \tilde{n}_{i}=n_{i}, \\
& \tilde{n}_{j}=n_{j}-n_{i} .
\end{aligned}
$$

The momenta of the particles in the two superparticles after collision are given by

$$
\begin{aligned}
& \tilde{\mathbf{V}}_{i} \tilde{m}_{i}=\mathbf{V}_{i} m_{i}+\mathbf{V}_{j} m_{j}, \\
& \tilde{\mathbf{V}}_{j} \tilde{m}_{j}=\mathbf{V}_{j} m_{j} .
\end{aligned}
$$

\section{APPENDIX B}

\section{Effect of Initial Condition on Collision in a Turbulent Environment}

The initial conditions are important for the collision. We tested three different initial conditions. Collision is triggered 1) in a randomly distributed superparticle field and the velocity of the flow is zero, 2) in a well-mixed particle field and the velocity of the flow is zero, and 3) in a

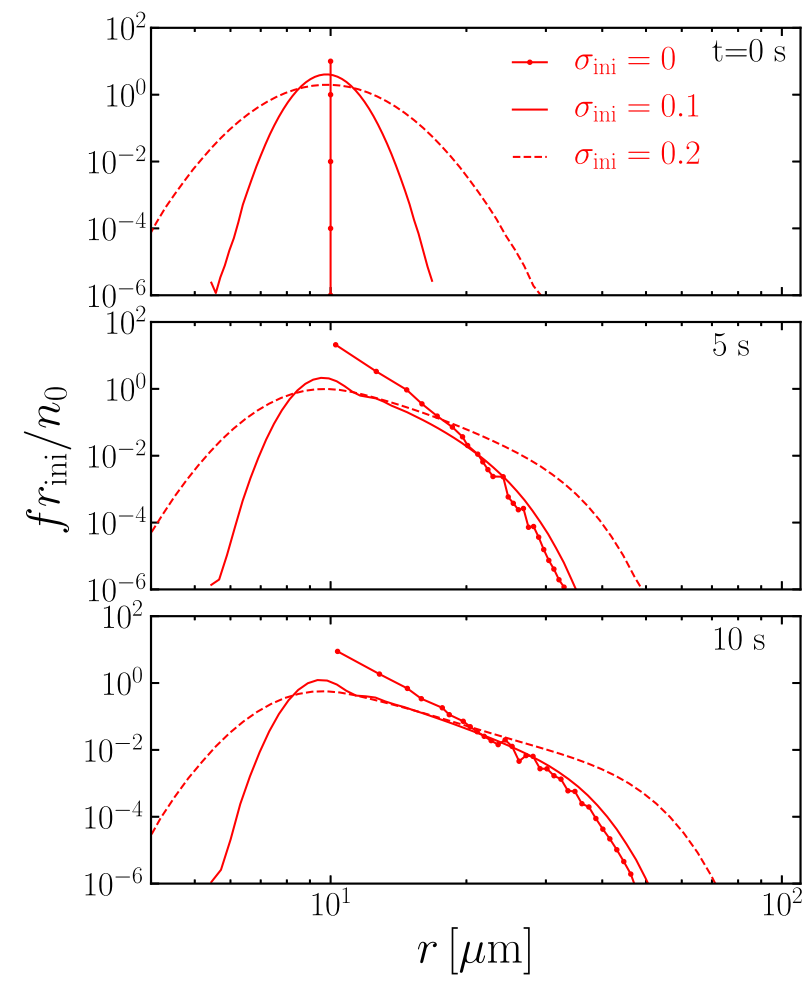

FIG. D1. Convergence of $\sigma_{\text {ini }}$. Collision is driven solely by turbulence; see run $\mathrm{B}$ for simulation details.

well-mixed particle field and the turbulence is well developed. To compare the time evolution of the size distribution for these three cases, we first define the normalized moments of the size distribution ( $\mathrm{Li}$ et al. 2017),

$$
a_{\zeta}=\left(\int_{0}^{\infty} f r^{\zeta} d r / \int_{0}^{\infty} f d r\right)^{1 / \zeta}
$$

where $\zeta$ is a positive integer. The mean radius $\bar{r}$ is given by $a_{1}$, the maximum radius is $\max (r)=a_{\infty}$, and the droplet mass is proportional to the third power of $a_{3}$. Parameter $a_{\zeta}$ can characterize the size distribution with simpler diagnostics. Figure B1 shows $a_{\zeta}$ for the three different initial conditions. It is obvious that the time evolution of $a_{\zeta}$ is independent from initial conditions. This can sufficiently save computational time.

\section{APPENDIX C}

\section{Statistical Convergence of the Number of Superparticles per Grid Cell in a Turbulent Environment}

As discussed in section 2, simulations with a massive number of superparticles are computationally 

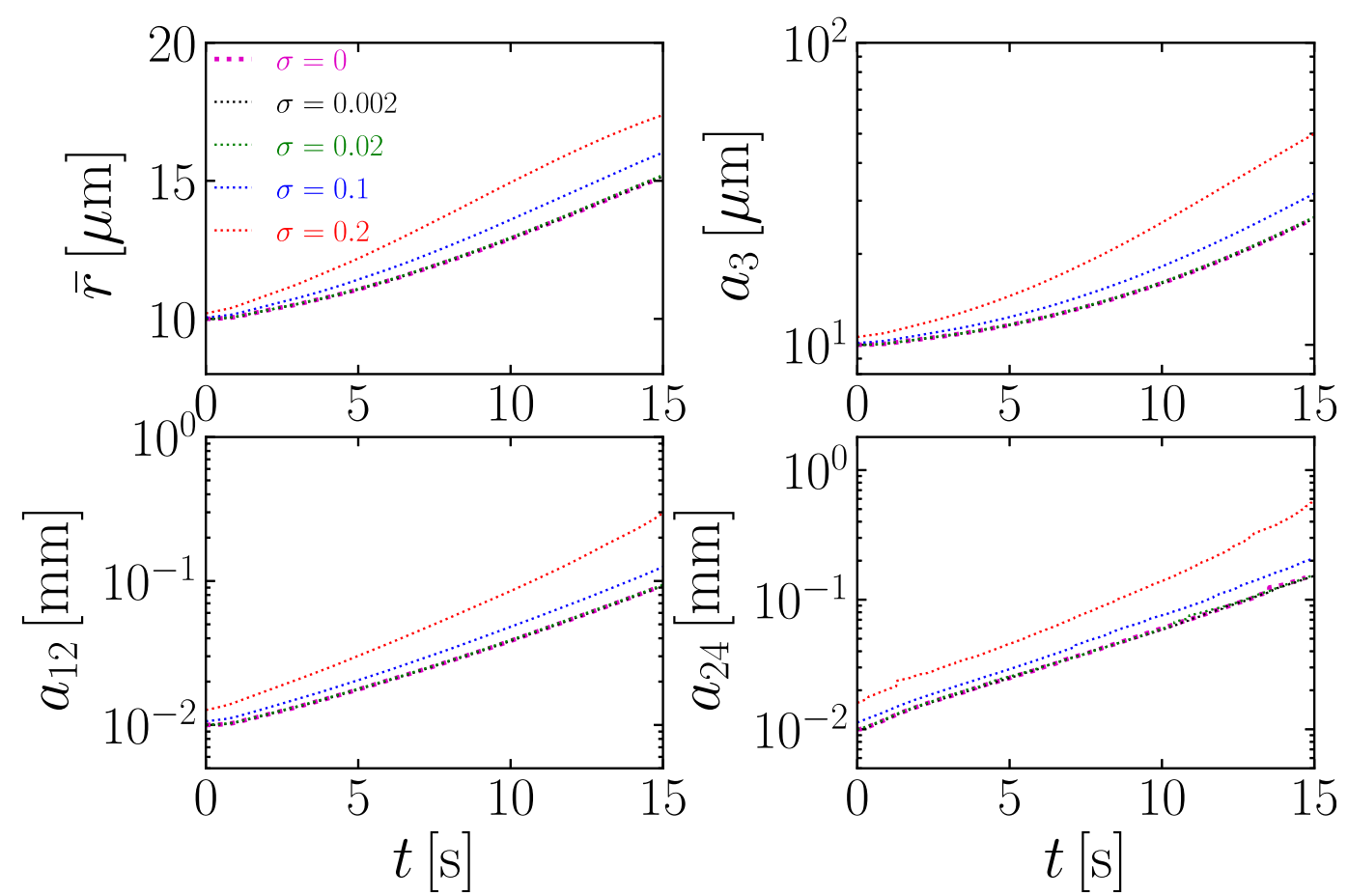

FIG. D2. Convergence of $\sigma_{\text {ini. }}$. Collision is driven by combined turbulence and gravity; see run B for simulation details.

costing. In Li et al. (2017), we found that the initial $N_{p} / N_{\text {grid }}$ converges at 4 when the collision is driven by gravity without turbulence. In the present study, $N_{\text {grid }}=512^{3}$. A value of $N_{p} / N_{\text {grid }}=4$ will result in $N_{p}=4 \times 512^{3}=536870912$, which will be very computationally demanding. This motivates us to restudy the convergence of $N_{p} / N_{\text {grid }}$ in high-Reynolds-number turbulence case instead of carrying the convergence study from pure gravity case to the turbulence case. As shown in Fig. $\mathrm{C} 1, N_{p} / N_{\text {grid }}$ converges at 0.5 . This could be because turbulence transports particles sufficiently.

\section{APPENDIX D}

\section{Convergence of the Initial Width $\sigma_{\text {ini }}$ in a Turbulent Environment with Gravity}

We first check the convergence of $\sigma_{\text {ini }}$ when collisions are driven solely by turbulence. As shown in Fig. D1, the time evolution of the size distribution almost converges at $\sigma_{\text {ini }}=0.1$. Compared with the case where the collision is driven by combined turbulence and gravity as demonstrated in Fig. 8, the tail of the size distribution is less sensitive to $\sigma_{\text {ini }}$. Next, we investigate the convergence of the width $\sigma_{\text {ini }}$ in Eq. (8) in a combined turbulence and gravity environment. Figure D 2 shows that $\sigma_{\text {ini }}$ converges at 0.02 . However, as we have discussed in section $3 \mathrm{~b}$, we choose $\sigma_{\text {ini }}=0$ for the combined turbulence and gravity case.

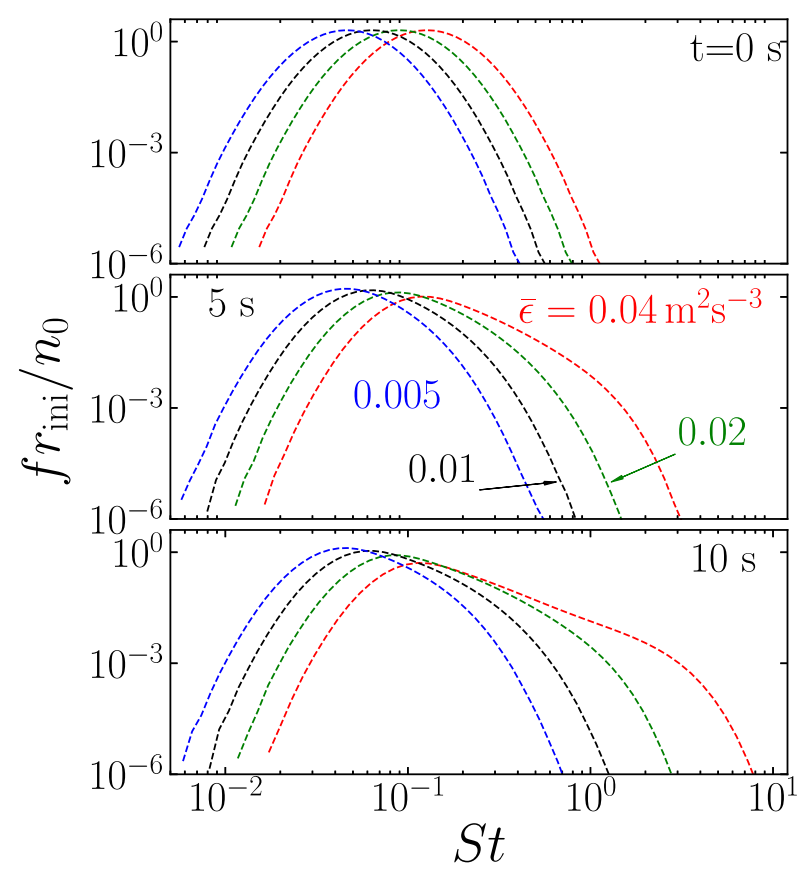

FIG. E1. Time evolution of the Stokes number distribution $\operatorname{St}(r, t)$ for different $\bar{\varepsilon}$ with fixed $\mathrm{Re}_{\lambda}$. Same simulations as in Fig. 2 . 

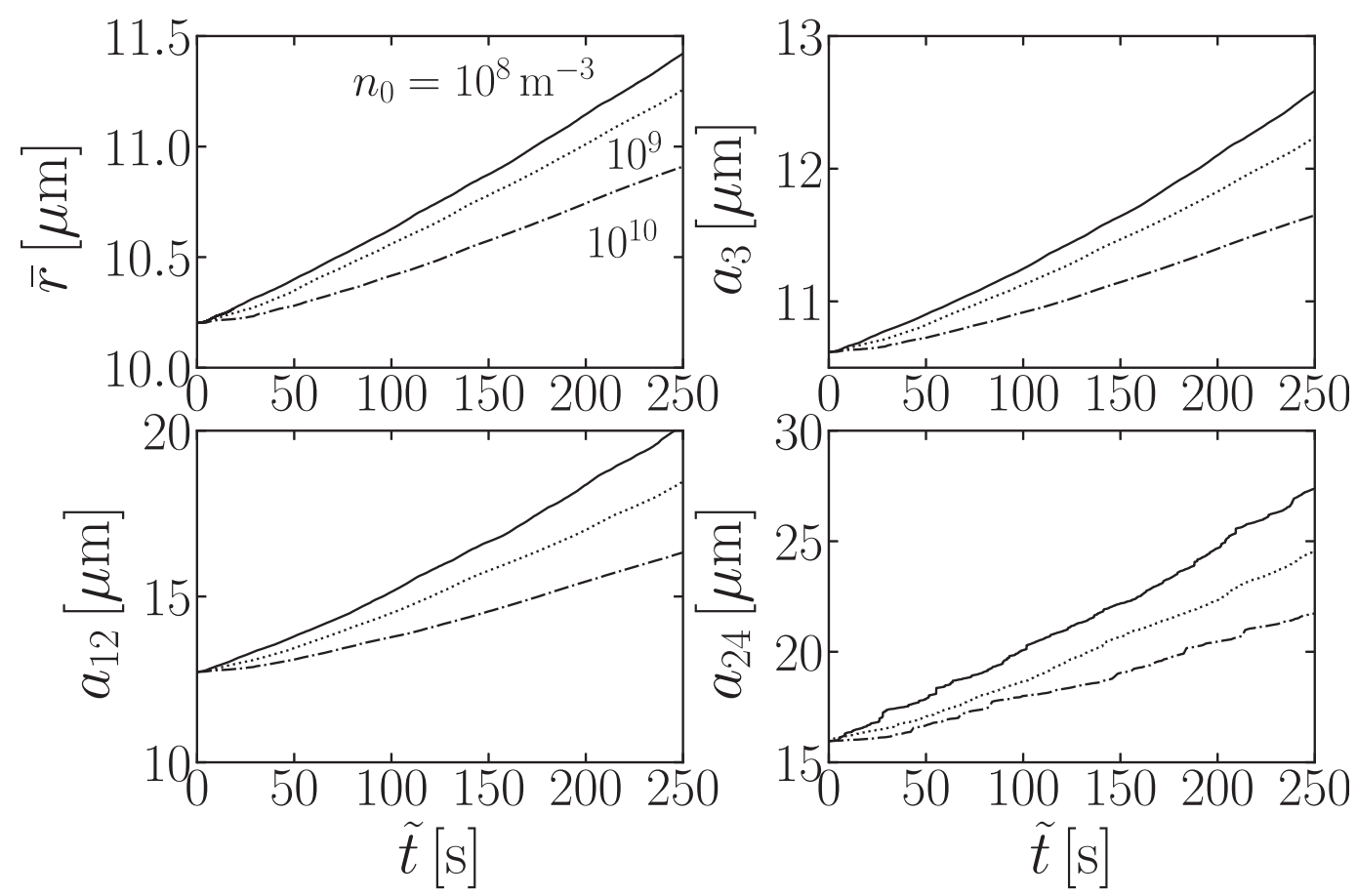

FIG. F1. Accuracy of the rescaling procedure of time in 2D turbulence. The size of the computational domain is $L=0.25 \mathrm{~m}$, the number of grid cells is $N_{\text {grid }}=512^{2}$, and the viscosity is $\nu=5 \times 10^{-4} \mathrm{~m}^{2} \mathrm{~s}^{-1}$, which gives a Taylor microscale Reynolds number of $\operatorname{Re}_{\lambda} \approx 106$ and an energy dissipation rate of $\bar{\varepsilon}=0.1 \mathrm{~m}^{2} \mathrm{~s}^{-3}$. The initial droplet size distribution is lognormal with $r_{\text {ini }}=10 \mu \mathrm{m}$ and $\sigma=0.2$. The number of the superparticles is $N_{p}=1.2 \times 10^{6}$ and the number of the grid cells is $N_{\text {grid }}=512^{2}$. Each simulation was conducted on 512 CPUs.

\section{APPENDIX E}

\section{Distribution of the Stokes Number}

Figure E1 shows the distribution function of Stokes numbers for the same simulations as in Fig. 2. Initially, the distribution of Stokes number shifts to the right with increasing $\bar{\varepsilon}$, which will trigger stronger collisional growth. At later times, when $\bar{\varepsilon}$ increases from 0.005 to $0.04 \mathrm{~m}^{2} \mathrm{~s}^{-3}$, the tail of the Stokes number distribution increases by more than an order of magnitude, which leads to an extension of about three orders of magnitude at $t=10 \mathrm{~s}$. This indicates that the collisional growth rate strongly depends on the Stokes number. Increasing $\bar{\varepsilon}$ results in a larger range of variations in the value of the Stokes number, thus enhancing the collisional growth.

\section{APPENDIX F}

\section{Rescaling of Time based on the Initial Number Density}

As explained at the end of section 2, simulating the collision-coalescence process of raindrop formation over realistic time scales is computationally demanding, so we adopt an initial number density of $n_{0}=10^{10} \mathrm{~m}^{-3}$ and rescale the simulation time to $\tilde{t}=t\left(n_{0} / n_{\text {ref }}\right)$. We check $a_{\zeta}$ for the rescaling in 2D turbulence. As shown in Fig. F1, larger values of $n_{0}$ result in smaller values of $a_{\zeta}$. However, the difference in $a_{24}$ is only about $5 \mu \mathrm{m}$ at $\tilde{t}=250 \mathrm{~s}$. This means that using $n_{0}=10^{10} \mathrm{~m}^{-3}$ does reasonably well represent the collisional growth.

\section{REFERENCES}

Andersson, B., K. Gustavsson, B. Mehlig, and M. Wilkinson, 2007: Advective collisions. Europhys. Lett., 80, 69001, https://doi.org/ 10.1209/0295-5075/80/69001.

Ayala, O., B. Rosa, and L.-P. Wang, 2008a: Effects of turbulence on the geometric collision rate of sedimenting droplets. Part 2. Theory and parameterization. New J. Phys., 10, 075016, https://doi.org/10.1088/1367-2630/10/7/075016.

,,--- , and W. W. Grabowski, 2008b: Effects of turbulence on the geometric collision rate of sedimenting droplets. Part 1. Results from direct numerical simulation. New J. Phys., 10, 075015, https://doi.org/10.1088/1367-2630/10/7/075015.

Beals, M. J., J. P. Fugal, R. A. Shaw, J. Lu, S. M. Spuler, and J. L. Stith, 2015: Holographic measurements of inhomogeneous cloud mixing at the centimeter scale. Science, 350, 87-90, https:// doi.org/10.1126/science.aab0751.

Bec, J., 2003: Fractal clustering of inertial particles in random flows. Phys. Fluids, 15, L81, https://doi.org/10.1063/1.1612500. 
—- A. Celani, M. Cencini, and S. Musacchio, 2005: Clustering and collisions in random flows. Phys. Fluids, 17, 073301, https://doi.org/10.1063/1.1940367.

Berry, E. X., and R. L. Reinhardt, 1974: An analysis of cloud drop growth by collection: Part I. Double distributions. J. Atmos. Sci., 31, 1814-1824, https://doi.org/10.1175/1520-0469(1974)031<1814: $\mathrm{AAOCDG}>2.0 . \mathrm{CO} ; 2$

Bird, G., 1978: Monte Carlo simulation of gas flows. Annu. Rev. Fluid Mech., 10, 11-31, https://doi.org/10.1146/annurev. fl.10.010178.000303.

_ 1981: Monte-Carlo simulation in an engineering context. Rarefied Gas Dynamics, Parts I and II, Progress in Astronautics and Aeronautics, AIAA, 239-255.

Brandenburg, A., 2001: The inverse cascade and nonlinear alphaeffect in simulations of isotropic helical hydromagnetic turbulence. Astrophys. J., 550, 824, https://doi.org/10.1086/319783.

Brilliantov, N., P. Krapivsky, A. Bodrova, F. Spahn, H. Hayakawa, V. Stadnichuk, and J. Schmidt, 2015: Size distribution of particles in Saturn's rings from aggregation and fragmentation. Proc. Natl. Acad. Sci. USA, 112, 9536-9541, https://doi.org/ 10.1073/pnas.1503957112.

Chandrakar, K. K., W. Cantrell, K. Chang, D. Ciochetto, D. Niedermeier, M. Ovchinnikov, R. A. Shaw, and F. Yang, 2016: Aerosol indirect effect from turbulence-induced broadening of cloud-droplet size distributions. Proc. Natl. Acad. Sci. USA, 113, 14243-14248, https://doi.org/10.1073/pnas.1612686113.

Chen, S., P. Bartello, M. Yau, P. Vaillancourt, and K. Zwijsen, 2016: Cloud droplet collisions in turbulent environment: Collision statistics and parameterization. J. Atmos. Sci., 73, 621-636, https://doi.org/10.1175/JAS-D-15-0203.1.

_ M. Yau, and P. Bartello, 2018: Turbulence effects of collision efficiency and broadening of droplet size distribution in cumulus clouds. J. Atmos. Sci., 75, 203-217, https://doi.org/ 10.1175/JAS-D-17-0123.1.

Chun, J., D. L. Koch, S. L. Rani, A. Ahluwalia, and L. R. Collins, 2005a: Clustering of aerosol particles in isotropic turbulence. J. Fluid Mech., 536, 219-251, https://doi.org/10.1017/ S0022112005004568.

,,,$--- \ldots$, and,$- 2005 \mathrm{~b}$ : Clustering of aerosol particles in isotropic turbulence. J. Fluid Mech., 536, 219-251, https://doi.org/10.1017/S0022112005004568.

Collins, L. R., and A. Keswani, 2004: Reynolds number scaling of particle clustering in turbulent aerosols. New J. Phys., 6, 119, https://doi.org/10.1088/1367-2630/6/1/119.

Devenish, B., and Coauthors, 2012: Droplet growth in warm turbulent clouds. Quart. J. Roy. Meteor. Soc., 138, 1401-1429, https://doi.org/10.1002/qj.1897.

Duncan, K., B. Mehlig, S. Östlund, and M. Wilkinson, 2005: Clustering in mixing flows. Phys. Rev. Lett., 95, 240602, https:// doi.org/10.1103/PhysRevLett.95.240602.

Dziekan, P., and H. Pawlowska, 2017: Stochastic coalescence in Lagrangian cloud microphysics. Atmos. Chem. Phys., 17, 13509 13 520, https://doi.org/10.5194/acp-17-13509-2017.

Elperin, T., N. Kleeorin, and I. Rogachevskii, 1996: Self-excitation of fluctuations of inertial particle concentration in turbulent fluid flow. Phys. Rev. Lett., 77, 5373-5376, https://doi.org/ 10.1103/PhysRevLett.77.5373.

, — - V. S. L'vov, I. Rogachevskii, and D. Sokoloff, 2002: Clustering instability of the spatial distribution of inertial particles in turbulent flows. Phys. Rev. E, 66, 036302, https:// doi.org/10.1103/PhysRevE.66.036302.

__, _- M. Liberman, and I. Rogachevskii, 2013: Tangling clustering instability for small particles in temperature stratified turbulence. Phys. Fluids, 25, 085104, https://doi.org/ 10.1063/1.4816643.

Falkovich, G., and A. Pumir, 2007: Sling effect in collisions of water droplets in turbulent clouds. J. Atmos. Sci., 64, 4497-4505, https://doi.org/10.1175/2007JAS2371.1.

A. Fouxon, and M. Stepanov, 2002: Acceleration of rain initiation by cloud turbulence. Nature, 419, 151-154, https:// doi.org/10.1038/nature00983.

Franklin, C. N., 2008: A warm rain microphysics parameterization that includes the effect of turbulence. J. Atmos. Sci., 65, 17951816, https://doi.org/10.1175/2007JAS2556.1.

—, P. A. Vaillancourt, M. Yau, and P. Bartello, 2005: Collision rates of cloud droplets in turbulent flow. J. Atmos. Sci., 62, 2451-2466, https://doi.org/10.1175/JAS3493.1.

Grabowski, W. W., and L.-P. Wang, 2013: Growth of cloud droplets in a turbulent environment. Annu. Rev. Fluid Mech., 45, 293324, https://doi.org/10.1146/annurev-fluid-011212-140750.

and G. C. Abade, 2017: Broadening of cloud droplet spectra through eddy hopping: Turbulent adiabatic parcel simulations. J. Atmos. Sci., 74, 1485-1493, https://doi.org/10.1175/ JAS-D-17-0043.1.

Grover, S., and H. Pruppacher, 1985: The effect of vertical turbulent fluctuations in the atmosphere on the collection of aerosol particles by cloud drops. J. Atmos. Sci., 42, 2305-2318, https:// doi.org/10.1175/1520-0469(1985)042<2305:TEOVTF>2.0.CO;2.

Gustavsson, K., and B. Mehlig, 2014: Relative velocities of inertial particles in turbulent aerosols. J. Turbul., 15, 34-69, https:// doi.org/10.1080/14685248.2013.875188.

— , and —, 2016a: Statistical model for collisions and recollisions of inertial particles in mixing flows. Eur. Phys. J. E, 39, 55, https://doi.org/10.1140/epje/i2016-16055-0.

_, and __ 2016b: Statistical models for spatial patterns of heavy particles in turbulence. Adv. Phys., 65, 1-57, https:// doi.org/10.1080/00018732.2016.1164490.

, - — and M. Wilkinson, 2008a: Collisions of particles advected in random flows. New J. Phys., 10, 075014, https:// doi.org/10.1088/1367-2630/10/7/075014.

$-, \ldots,-$, and V. Uski, 2008b: Variable-range projection model for turbulence-driven collisions. Phys. Rev. Lett., 101, 174503, https://doi.org/10.1103/PhysRevLett.101.174503.

_ S. Vajedi, and B. Mehlig, 2014: Clustering of particles falling in a turbulent flow. Phys. Rev. Lett., 112, 214501, https:// doi.org/10.1103/PhysRevLett.112.214501.

Ireland, P. J., A. D. Bragg, and L. R. Collins, 2016a: The effect of Reynolds number on inertial particle dynamics in isotropic turbulence. Part 1. Simulations without gravitational effects. J. Fluid Mech., 796, 617-658, https://doi.org/10.1017/ jfm.2016.238.

_ — - and _ 2016b: The effect of Reynolds number on inertial particle dynamics in isotropic turbulence. Part 2. Simulations with gravitational effects. J. Fluid Mech., 796, 659-711, https://doi.org/10.1017/jfm.2016.227.

Johansen, A., and M. Lambrechts, 2017: Forming planets via pebble accretion. Annu. Rev. Earth Planet. Sci., 45, 359-387, https://doi.org/10.1146/annurev-earth-063016-020226.

— A. A. Youdin, and Y. Lithwick, 2012: Adding particle collisions to the formation of asteroids and Kuiper belt objects via streaming instabilities. Astron. Astrophys., 537, A125, https:// doi.org/10.1051/0004-6361/201117701.

Jorgensen, W. L., J. Chandrasekhar, J. D. Madura, R. W. Impey, and M. L. Klein, 1983: Comparison of simple potential functions for simulating liquid water. J. Chem. Phys., 79, 926-935, https://doi.org/10.1063/1.445869. 
Kostinski, A. B., and R. A. Shaw, 2001: Scale-dependent droplet clustering in turbulent clouds. J. Fluid Mech., 434, 389-398, https://doi.org/10.1017/S0022112001004001.

$\longrightarrow$, and —, 2005: Fluctuations and luck in droplet growth by coalescence. Bull. Amer. Meteor. Soc., 86, 235-244, https:// doi.org/10.1175/BAMS-86-2-235.

Koziol, A. S., and H. Leighton, 1996: The effect of turbulence on the collision rates of small cloud drops. J. Atmos. Sci., 53, 1910-1920, https://doi.org/10.1175/1520-0469(1996)053<1910: TEOTOT $>2.0 . \mathrm{CO} ; 2$.

Lamb, D., and J. Verlinde, 2011: Warm clouds. Physics and Chemistry of Clouds, Cambridge University Press, 433-456.

Li, X.-Y., A. Brandenburg, N. E. L. Haugen, and G. Svensson, 2017: Eulerian and Lagrangian approaches to multidimensional condensation and collection. J. Adv. Model. Earth Syst., 9, 1116-1137, https://doi.org/10.1002/2017MS000930.

Marchioli, C., and Coauthors, 2008: Statistics of particle dispersion in direct numerical simulations of wall-bounded turbulence: Results of an international collaborative benchmark test. Int. J. Multiph. Flow, 34, 879-893, https://doi.org/10.1016/ j.ijmultiphaseflow.2008.01.009.

Mathis, J. S., W. Rumpl, and K. H. Nordsieck, 1977: The size distribution of interstellar grains. Astrophys. J., 217, 425-433, https://doi.org/10.1086/155591.

Maxey, M., 1987: The gravitational settling of aerosol particles in homogeneous turbulence and random flow fields. J. Fluid Mech., 174, 441-465, https://doi.org/10.1017/S0022112087000193.

Mehlig, B., M. Wilkinson, and V. Uski, 2007: Colliding particles in highly turbulent flows. Phys. Fluids, 19, 098107, https://doi.org/ 10.1063/1.2768931.

Meibohm, J., L. Pistone, K. Gustavsson, and B. Mehlig, 2017: Relative velocities in bidisperse turbulent suspensions. Phys. Rev. E, 96, 061102, https://doi.org/10.1103/PhysRevE.96.061102.

Miles, N. L., J. Verlinde, and E. E. Clothiaux, 2000: Cloud droplet size distributions in low-level stratiform clouds. J. Atmos. Sci., 57, 295-311, https://doi.org/10.1175/1520-0469(2000)057<0295: CDSDIL $>2.0 . \mathrm{CO} ; 2$.

Nenes, A., and J. H. Seinfeld, 2003: Parameterization of cloud droplet formation in global climate models. J. Geophys. Res., 108, 4415, https://doi.org/10.1029/2002JD002911.

Ohno, K., and S. Okuzumi, 2017: A condensation-coalescence cloud model for exoplanetary atmospheres: Formulation and test applications to terrestrial and Jovian clouds. Astrophys. J., 835, 261, https://doi.org/10.3847/1538-4357/835/ $2 / 261$.

Onishi, R., and A. Seifert, 2016: Reynolds-number dependence of turbulence enhancement on collision growth. Atmos. Chem. Phys., 16, 12 441-12 455, https://doi.org/10.5194/ acp-16-12441-2016.

Perrin, V. E., and H. J. Jonker, 2015: Lagrangian droplet dynamics in the subsiding shell of a cloud using direct numerical simulations. J. Atmos. Sci., 72, 4015-4028, https://doi.org/10.1175/ JAS-D-15-0045.1.

Pinsky, M., and A. Khain, 2004: Collisions of small drops in a turbulent flow. Part II: Effects of flow accelerations. J. Atmos. Sci., 61, 1926-1939, https://doi.org/10.1175/1520-0469(2004) $061<1926$ :COSDIA $>2.0 . \mathrm{CO} ; 2$.

,$- \ldots$, and M. Shapiro, 2007: Collisions of cloud droplets in a turbulent flow. Part IV: Droplet hydrodynamic interaction. J. Atmos. Sci., 64, 2462-2482, https://doi.org/10.1175/ JAS3952.1.

,-- , and H. Krugliak, 2008: Collisions of cloud droplets in a turbulent flow. Part V: Application of detailed tables of turbulent collision rate enhancement to simulation of droplet spectra evolution. J. Atmos. Sci., 65, 357-374, https://doi.org/ 10.1175/2007JAS2358.1.

Pope, S., 2000: Turbulent Flows. Cambridge University Press, 802 pp. Pruppacher, H. R., J. D. Klett, and P. K. Wang, 1998: Microphysics of Clouds and Precipitation. Taylor and Francis, $954 \mathrm{pp}$.

Pumir, A., and M. Wilkinson, 2016: Collisional aggregation due to turbulence. Annu. Rev. Condens. Matter Phys., 7, 141-170, https://doi.org/10.1146/annurev-conmatphys-031115-011538.

Reade, W. C., and L. R. Collins, 2000: Effect of preferential concentration on turbulent collision rates. Phys. Fluids, 12, 25302540, https://doi.org/10.1063/1.1288515.

Reuter, G., R. De Villiers, and Y. Yavin, 1988: The collection kernel for two falling cloud drops subjected to random perturbations in a turbulent air flow: A stochastic model. J. Atmos. Sci., 45, 765-773, https://doi.org/10.1175/1520-0469(1988) 045<0765:TCKFTF $>2.0 . \mathrm{CO} ; 2$.

Riemer, N., and A. Wexler, 2005: Droplets to drops by turbulent coagulation. J. Atmos. Sci., 62, 1962-1975, https://doi.org/ 10.1175/JAS3431.1.

Rosa, B., H. Parishani, O. Ayala, W. W. Grabowski, and L.-P. Wang, 2013: Kinematic and dynamic collision statistics of cloud droplets from high-resolution simulations. New J. Phys., 15, 045032, https://doi.org/10.1088/1367-2630/15/4/ 045032.

Saffman, P. G., and J. S. Turner, 1956: On the collision of drops in turbulent clouds. J. Fluid Mech., 1, 16-30, https://doi.org/ 10.1017/S0022112056000020.

Saito, I., and T. Gotoh, 2018: Turbulence and cloud droplets in cumulus clouds. New J. Phys., 20, 023001, https://doi.org/ 10.1088/1367-2630/aaa229.

Salazar, J. P., J. De Jong, L. Cao, S. H. Woodward, H. Meng, and L. R. Collins, 2008: Experimental and numerical investigation of inertial particle clustering in isotropic turbulence. J. Fluid Mech., 600, 245-256, https://doi.org/10.1017/ S0022112008000372.

Sardina, G., F. Picano, L. Brandt, and R. Caballero, 2015: Continuous growth of droplet size variance due to condensation in turbulent clouds. Phys. Rev. Lett., 115, 184501, https://doi.org/ 10.1103/PhysRevLett.115.184501.

Schiller, L., and A. Naumann, 1933: Fundamental calculations in gravitational processing. Z. Ver. Dtsch. Ing., 77, 318-320.

Seinfeld, J. H., and S. N. Pandis, 2016: Atmospheric Chemistry and Physics: From Air Pollution to Climate Change. John Wiley \& Sons, $1232 \mathrm{pp}$.

Shaw, R. A., 2003: Particle-turbulence interactions in atmospheric clouds. Annu. Rev. Fluid Mech., 35, 183-227, https://doi.org/ 10.1146/annurev.fluid.35.101101.161125.

—_ W. C. Reade, L. R. Collins, and J. Verlinde, 1998: Preferential concentration of cloud droplets by turbulence: Effects on the early evolution of cumulus cloud droplet spectra. J. Atmos. Sci., 55, 1965-1976, https://doi.org/10.1175/ 1520-0469(1998)055<1965:PCOCDB > 2.0.CO;2.

Shima, S., K. Kusano, A. Kawano, T. Sugiyama, and S. Kawahara, 2009: The super-droplet method for the numerical simulation of clouds and precipitation: A particle-based and probabilistic microphysics model coupled with a non-hydrostatic model. Quart. J. Roy. Meteor. Soc., 135, 1307-1320, https://doi.org/ 10.1002/qj.441.

Sundaram, S., and L. R. Collins, 1997a: Collision statistics in an isotropic particle-laden turbulent suspension. J. Fluid Mech., 335, 75-109, https://doi.org/10.1017/S0022112096004454. 
and - , 1997b: Collision statistics in an isotropic particleladen turbulent suspension. Part 1. Direct numerical simulations. J. Fluid Mech., 335, 75-109, https://doi.org/10.1017/ S0022112096004454.

Telford, J. W., 1955: A new aspect of coalescence theory. J. Meteor., 12, 436-444, https://doi.org/10.1175/1520-0469(1955)012<0436: ANAOCT $>2.0 . \mathrm{CO} ; 2$

Veysey, J., II, and N. Goldenfeld, 2007: Simple viscous flows: From boundary layers to the renormalization group. Rev. Mod. Phys., 79, 883-927, https://doi.org/10.1103/RevModPhys.79.883.

Völk, H. J., F. C. Jones, G. E. Morfill, and S. Röser, 1980: Collisions between grains in a turbulent gas. Astron. Astrophys., 85, 316-325.

Voßkuhle, M., A. Pumir, E. Lévêque, and M. Wilkinson, 2014: Prevalence of the sling effect for enhancing collision rates in turbulent suspensions. J. Fluid Mech., 749, 841-852, https:// doi.org/10.1017/jfm.2014.259.

Wang, L.-P., and W. W. Grabowski, 2009: The role of air turbulence in warm rain initiation. Atmos. Sci. Lett., 10,1-8, https:// doi.org/10.1002/asl.210.

_ A. S. Wexler, and Y. Zhou, 1998: Statistical mechanical descriptions of turbulent coagulation. Phys. Fluids, 10, 26472651, https://doi.org/10.1063/1.869777.

, O. Ayala, S. E. Kasprzak, and W. W. Grabowski, 2005: Theoretical formulation of collision rate and collision efficiency of hydrodynamically interacting cloud droplets in turbulent atmosphere. J. Atmos. Sci., 62, 2433-2450, https:// doi.org/10.1175/JAS3492.1.

, - Y. Xue, and W. W. Grabowski, 2006: Comments on "droplets to drops by turbulent coagulation." J. Atmos. Sci., 63, 2397-2401, https://doi.org/10.1175/JAS3750.1.

- - - and W. Grabowski, 2007: Effects of aerodynamic interactions on the motion of heavy particles in a bidisperse suspension. J. Turbul., 8, N25, https://doi.org/10.1080/ 14685240701233426.

Wilkinson, M., 2016: Large deviation analysis of rapid onset of rain showers. Phys. Rev. Lett., 116, 018501, https://doi.org/10.1103/ PhysRevLett.116.018501.

— B. Mehlig, and V. Bezuglyy, 2006: Caustic activation of rain showers. Phys. Rev. Lett., 97, 048501, https://doi.org/10.1103/ PhysRevLett.97.048501.

Woittiez, E. J. P., H. J. J. Jonker, and L. M. Portela, 2009: On the combined effects of turbulence and gravity on droplet collisions in clouds: A numerical study. J. Atmos. Sci., 66, 19261943, https://doi.org/10.1175/2005JAS2669.1.

Xue, Y., L.-P. Wang, and W. W. Grabowski, 2008: Growth of cloud droplets by turbulent collision-coalescence. J. Atmos. Sci., 65 , 331-356, https://doi.org/10.1175/2007JAS2406.1.

Zsom, A., and C. P. Dullemond, 2008: A representative particle approach to coagulation and fragmentation of dust aggregates and fluid droplets. Astron. Astrophys., 489, 931-941, https:// doi.org/10.1051/0004-6361:200809921. 